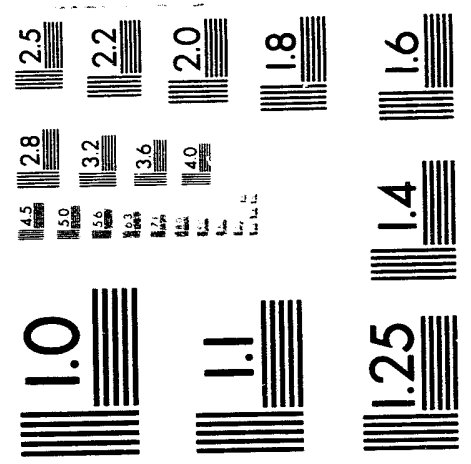



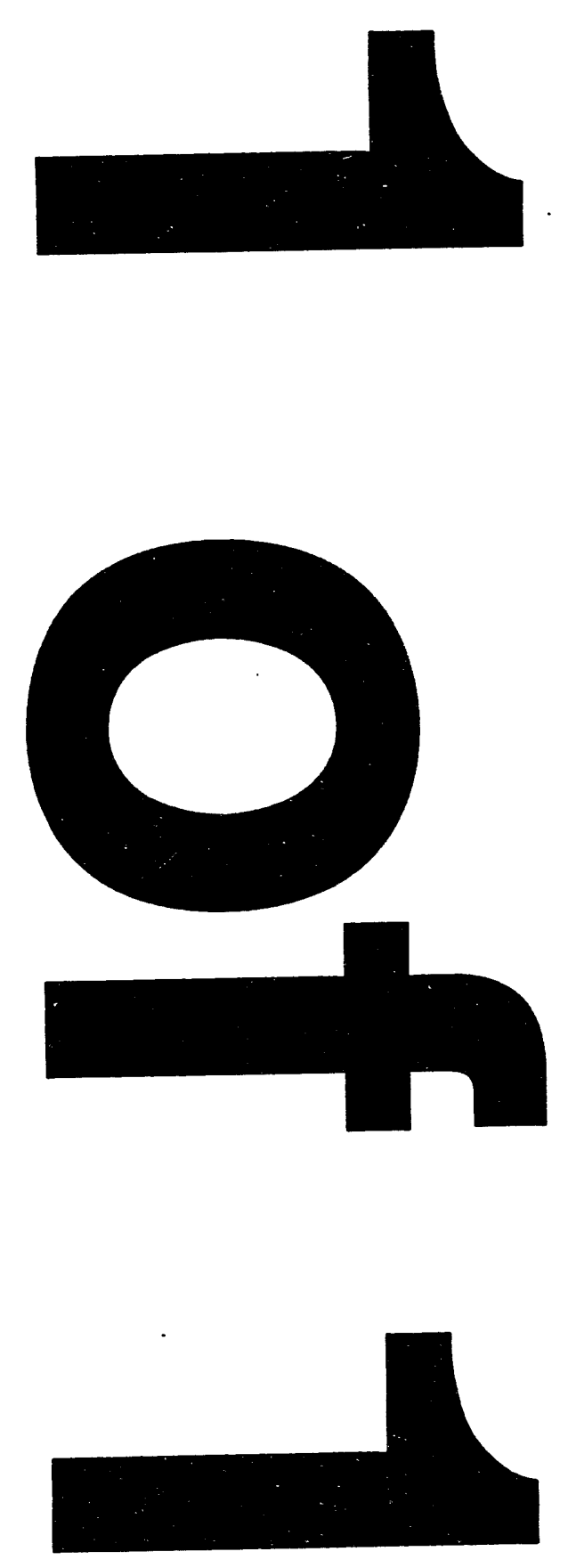


\title{
SAND94-0314
}

\section{FWP EXECUTIVE SUMMARIES}

BASIC ENERGY SCIENCES

MATERIALS SCIENCES PROGRAMS

(SNL/NM)

\author{
G. A. Samara \\ Program Manager
}

Sandia National Laboratories

Albuquerque, New Mexico

January 1994 


\section{Table of Contents}

I. Budget $\ldots \ldots \ldots \ldots \ldots \ldots \ldots \ldots \ldots \ldots \ldots \ldots \ldots \ldots \ldots \ldots \ldots \ldots \ldots \ldots \ldots$

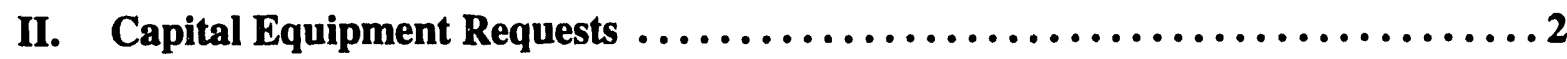

III. General Programmatic Overview and Institutional Issues $\ldots \ldots \ldots \ldots \ldots \ldots \ldots$

IV. The DOE Center of Excellence For The Synthesis and

Processing of Advanced Materials . . . . . . . . . . . . . . . . . . 10

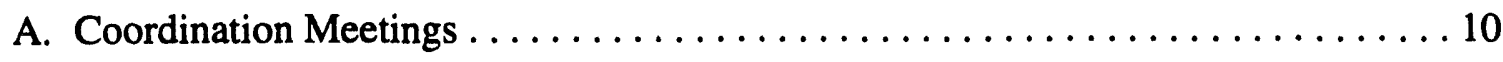

B. The Center's Industrial Steering Group $\ldots \ldots \ldots \ldots \ldots \ldots \ldots \ldots \ldots \ldots$

1. ISG Membership. . . . . . . . . . . . . . . . . . . . . . 11

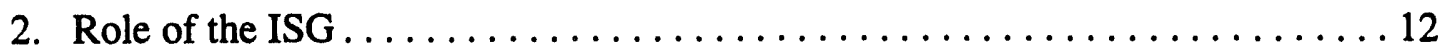

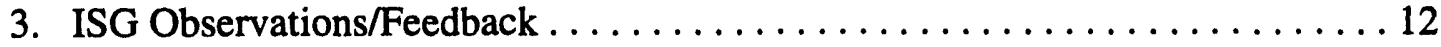

V. Industrial Interactions and Technology Transfer $\ldots \ldots \ldots \ldots \ldots \ldots \ldots \ldots$

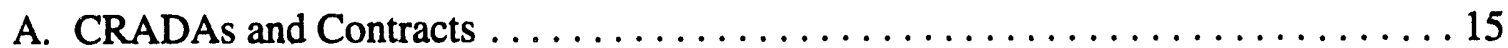

B. Other Industrial Interactions $\ldots \ldots \ldots \ldots \ldots \ldots \ldots \ldots \ldots \ldots \ldots \ldots \ldots \ldots \ldots \ldots \ldots \ldots$

VI. Research Program Summaries.......................... 20

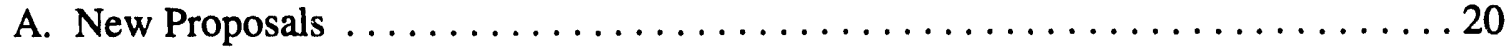

1. New Initiative Proposal

-Wetting and Flow of Liquid Metals and

Amorphous Ceramics at Solid Interfaces .............. 21

2. Other Proposal

-Ceramic Toughening by Crack-Interface Bridging . . . . . . . . 24

B. Existing Programs. . . . . . . . . . . . . . . . . . . . . . . 26

1. Significant Accomplishments

-Ambient Temperature/Pressure Processing of Aerogels . . . . . . . . . . 27

-Broadband Light Emission From Fractal Quantum Wells . . . . . . . . . 28 
-Phonon-Mediated Attraction Between Large Bipolarons:

Formation of a Bipolaronic Liquid. . . . . . . . . . . . . . . . 29

-New, Stronger Mechanism for Impurity Gettering in Silicon

Attracts Industry and Government Interest. . . . . . . . . . . . 30

-Interfacial-Force Microscopy Probes the Mechanical Properties of Single, Nanometer-Size Metal Grains . . . . . . . . . . . . . 31

-Direct Observation of Boron-Dopant Induced Effects on SiO2 CVD ... . 32

-Band Structure Effects in Fullerene-Based Superconductors .......... 33

-A New Understanding of Electron Cyclotron Resonance Plasmas

Permits Controlled Hydrogen Treatment of Electronic Materials . . . . . 34

-Unified Model of Roughening Instability and Surface

Evolution During Low Energy Ion Bombardment ............. 35

-Direct Observation of Substitutional-Atom Trapping on a Metal Surface . . 36

-Infrared Spectroscopy of Adsorbates on GaAs Surfaces............ 37

2. Research Summaries.............................. 38

-Physics and Chemistry of Ceramics ...................... 39

-Atomic Level Science of Adhesion..................... 42

-Energetic-Particle Synthesis and Science of Materials ............. 44

-Advanced Growth Techniques and the Science of Epitaxy .......... 46

-Artificially-Structured Semiconductors. .................. 48

-Physics and Chemistry of Novel Superconductors ............ 50

-Tailored Surfaces and Interfaces for Materials Applications ......... 52

-Boron-Rich Solids ............................ 54

-CVD Sciences . . . . . . . . . . . . . . . . . . . . . . . . 56

-Synthesis and Processing of Nanoclusters for Energy Applications. . . . . . 59 


\section{Budget}

Basic Energy Sciences/Materials Sciences Funding for FY93-96

Sandia National Laboratories - Albuquerque, New Mexico

\begin{tabular}{|c|c|c|c|c|c|}
\hline $\begin{array}{l}\text { Active } \\
\text { Programs }\end{array}$ & Title & $\begin{array}{c}\text { AFP } \\
\text { FY93 } \\
(\$ K)\end{array}$ & $\begin{array}{l}\text { AFP } \\
\text { FY94 } \\
\text { (\$K) }\end{array}$ & $\begin{array}{c}\text { Projected } \\
\text { FY95 } \\
\text { (\$K) }\end{array}$ & $\begin{array}{c}\text { Projected } \\
\text { FY96 } \\
\text { (\$K) }\end{array}$ \\
\hline KC020101 & Physics \& Chemistry of Ceramics & 1,034 & 979 & 1,028 & 1,079 \\
\hline KC020102 & Science of Adhesion & 523 & 494 & 519 & 545 \\
\hline KC020103 & Energetic Particle Synthesis & 882 & 835 & 877 & 921 \\
\hline KC020103 & Advanced Growth & 389 & 368 & 386 & 406 \\
\hline KC020105 & $\begin{array}{l}\text { Artificially-Structured } \\
\text { Semiconductors }\end{array}$ & 375 & 354 & 372 & 390 \\
\hline KC020105 & Ceramic Hi T Superconductors & 74 & 71 & 75 & 78 \\
\hline KC020202 & $\begin{array}{l}\text { Tailored Surfaces for Materials } \\
\text { Application }\end{array}$ & 632 & 600 & 630 & 662 \\
\hline KC020202 & $\begin{array}{l}\text { Physics \& Chemistry of Novel } \\
\text { Superconductors }\end{array}$ & 340 & 328 & 344 & 362 \\
\hline KC020202 & Synthesis \& Processing Center & 10 & 0 & 0 & 0 \\
\hline KC020205 & High Temperature Superconductivity & 262 & 247 & 259 & 272 \\
\hline KC020205 & Boron-Rich Solids & 503 & 475 & 499 & 524 \\
\hline KC020205 & Synthesis \& Processing Center & & 200 & 210 & 221 \\
\hline KC020303 & CVD Sciences & 792 & 700 & 735 & 772 \\
\hline \multirow[t]{2}{*}{ KC020303 } & Nanoclusters & & $\underline{250}$ & 263 & 276 \\
\hline & TOTALS & 5,806 & 5,901 & 6,197 & 6,508 \\
\hline Equipment & & 435 & 435 & 500 & 500 \\
\hline Award & & 150 & 100 & & \\
\hline
\end{tabular}

New Initiative Proposal

Wetting and Flow of Liquid Metals and Amorphous Ceramics at Solid Interfaces .....650K

Other Proposal

Ceramic Toughening by Check Interface Bridging $\ldots \ldots \ldots \ldots \ldots \ldots \ldots \ldots \mathrm{K}^{*}$

*These activities proposed for FY96 start ups could start in FY95 if funds become available. 


\section{Capital Equipment Requests}

\section{A. Mid Year (FY94) Requests (In priority Order)}

1. CCD Camera System for TEM

$\$ 130 K$

A charge-coupled device (CCD) camera system is needed to improve our TEM capabilities used to provide key microstructural information for three BES projects (Energetic Particle Synthesis, Artificially Structured Semiconductors, and Advanced Growth). Two CCD camera units are involved. First, a slow-scan CCD camera is needed to provide near-photographic imaging in livetime. This device can improve stigmation for high-resolution imaging and allow images taken with low intensities, e.g., dark-field, weak beam images and low-intensity diffraction patterns, to be viewed during the imaging session. Moreover, the digitized images can be computer processed to enhance contrast or take fourier transforms. Second, a TV-rate, wide-angle camera is needed to allow investigation over larger areas at lower magnifications; its images would be coupled to digitizing interfaces to allow computer processing. This new capability is based on advances in imaging technology and will enable a significant enhancement in the efficiency of operations (immediate high sensitivity images to adjust for proper exposure as well as to further investigate new, low-intensity information without waiting for 1 day cycle time via chemical processing of negatives). Also it will allow for new types of studies such as diffraction from internal surfaces and interfaces (nanocavities, ordered precipitates, fined grained structures, etc.), and for quantifying the intensity of diffraction as in ordered structures.

2. Elemental Analysis Instrument

$\$ 65 K$

New elemental analysis equipment is needed for our thin films program. An essential part of determining identity and purity of new compounds is elemental analysis. Currently the in-house facilities at Sandia can not handle air sensitive compounds at an acceptable level. In addition, In-lab analysis will speed up compound identification and progress of our program. Delay and costs with contracted analysis preclude use of external sources of analysis.

3. Mode-Locked Laser

\$50K

A mode-locked, tunable Titanium Sapphire laser is needed for studying optical properties of semiconductor structures. This laser will allow ultrashort, wavelength tunable laser pulses to be produced. The pulse width is about 100 femtoseconds which is short enough to study electron-hole relaxation in higher lying energy bands. Such relaxation studies are critical to the development of new fractal quantum well materials. These materials have tailorable absorption and have high efficiency, broadband emission. These artificial semiconductors have potential for photovoltaic applications. Without this capability, the development of these new materials will be slowed. 


\section{B. Major Equipment Needs for FY95 (In Priority Order)}

1. Injection-Stabilized Tunable Laser System

\$250K

A single-frequency laser source with non-linear doubling and mixing capabilities is necessary for the development of advanced in situ measurement techniques for studying complex chemistry in CVD and MOCVD processes. Advances in laser technology that have occurred over the last 6 years have led to commercially available lasers with significantly improved pulse-to-pulse reproducibility and narrower, better controlled spectral characteristics. These new lasers now make it practical to introduce more sophisticated laser spectroscopies, for example resonance Raman spectroscopy, multiphoton ionization spectroscopy or surface sum-frequency generation, into applied spectroscopy studies. Previously these techniques require such tour de force efforts that they were largely limited to proof- of-principle experiments in laser laboratories and were rarely used as routine probes of chemically reacting systems.

A major goal of the CVD Sciences program is to develop new tools for investigating the fundamental chemistry underlying CVD processes. In the past we have relied on fairly straight-forward optical probes like conventional Raman spectroscopy or laser-induced fluorescence (LIF) to identify and spatially resolve concentrations of important gas-phase species. Recently a rather sophisticated combined molecular beam/LIF technique dubbed IRIS (Imaging of Radicals Interacting with Surfaces) was developed under this project to provide a means for measuring the reactive sticking coefficients of radical species. At present, all of these techniques are severely limited, both in signal-to-noise and range of applicability, by the quality and performance characteristics of the lasers involved. This is most clearly evident in the wavelength ranges that can be accessed with our existing sources (all of which have been borrowed from other organizations or projects).

A new, state-of-the-art laser system with extended UV capabilities and more stable frequency and intensity characteristics will have a significant impact on the BES/MS CVD Sciences program by (1) broadening the range of molecules accessible with existing LIF and Raman techniques, by (2) improving the sensitivity, detectivity and S/N of our measurements, decreasing data acquisition times and by (3) enabling the exploration of more sophisticated multiphoton and/or multi-wavelength spectroscopies for detecting and monitoring chemical species that are currently inaccessible by traditional spectroscopies.

It should be noted that our existing laser systems (and parts for them) are no longer being produced by the manufacturer, and thus our ability to maintain these lasers is in jeopardy. To date we have been able to cannibalize other lasers of the same vintage for repair parts, however we cannot count on this approach indefinitely. Increasing down-time also continues to be a problem affecting productivity of staff time. Even if our existing lasers do continue to function, there remains, as discussed above, a significant limitation on our ability to advance the research and development of new optical in situ diagnostic probes. 
Justification - Imaging techniques have revolutionized materials science. Whereas early microscopies were used only as analytical tools, the development of the Transmission Electron Microscope (TEM) enabled researchers to first observe and then connect defect structures with important materials properties. Subsequently, the Scanning Tunneling Microscope (STM), has enabled us to "see" how materials grow and nanostructures form, atom by atom, thereby helping to establish the atomistic models needed to predict and control material growth.

However, solutions to many important problems involving microstructure formation and stability remain elusive. Examples include high-temperature thin film stability, delamination, oxidation and corrosion, defect propagation, and materials synthesis in chemically harsh environments such as CVD. Invented only three years ago by a European physicist, the Low Energy Electron Microscope (LEEM) is an instrument which is ideally suited to addressing these materials problems. Only three such instruments currently exist in the world. Because the LEEM is based upon precise electron optics rather than tunneling, nanometer-scale, two-dimensional images of microstructural changes can be obtained in real time under rigorous sample conditions. No other technique has this combination of high spatial resolution, sample flexibility, and continuous real-time imaging.

We will integrate the LEEM capability into the BES Materials Science programs at Sandia. We will study the growth mechanisms, microstructures, and stability of materials and ultra thin coatings during thermal and chemical processing. Predictive models for microstructural processes based upon atomistic theory, laboratory spectroscopies, and STM will be tested directly by the quantitative LEEM results. Thus the LEEM is the research tool that will enable us to advance our established multi-technique approach and to understand materials properties at a new level of complexity. Because the LEEM is an uncommon and powerful research tool, we envision its use in Sandia technology transfer programs as well, thereby enabling our industrial CRADA partners access to this advanced instrumentation.

If not purchased - Without the LEEM, the BES Materials Science programs at Sandia will not have the capability to image and quantify dynamic surface and interface processes and chemical reactions at the nanometer level--the forefront of microelectronics and materials research. Static model systems provide little insight into kinetic mechanisms and energetic of nucleation and growth, interdiffusion, wetting and spreading, and corrosion. Technology transfer industrial partners will not be able to access a state-of-the-art miscroscopy ideally suited for advanced materials synthesis and stability research. 


\section{General Programmatic Overview and Institutional Issues}

The goals of the BES Materials Sciences Program at Sandia are:

- Perform basic, forefront interdisciplinary research that also demonstrates relevance of basic science to technology.

- Choose programs which support the National DOE Strategy and are complementary to Sandia's multiprogram laboratory mission.

- Perform research in a setting which enhances technological impact because of Sandia's spectrum of coordinated basic research, applied research and development engineering.

- Take advantage of a complex of large, capital-intensive research facilities not usually found at universities.

Our BES Materials Sciences Program has the central theme of Scientifically Tailored Materials. The major objective of this program is to combine Sandia's expertise and capabilities in the areas of solid state sciences, advanced atomic-level diagnostics and materials synthesis and processing science to produce new clas s:s of tailored materials for U.S. energy applications, for industrial applications and for critical defense needs.

Current research in this program at SNL/NM includes the physics and chemistry of ceramics synthesis and processing, the use of energetic particles for the synthesis and study of materials, high-temperature and organic superconductors, tailored surfaces and interfaces for materials applications, chemical vapor deposition sciences, strained-layer and artificially structured semiconductors, advanced growth techniques for improved semiconductor structures, boron-rich solids and atomic level science of interfacial adhesion. Considerable synergism exists among these program elements, and most of them share a number of common themes based on either the science or the use of common synthesis and processing approaches. These themes are: Synthesis and Processing, Epitaxial Growth, Surfaces and Interfaces, Energetic Beams and High Tc Superconductivity. Many of the technical activities of the program have been incorporated into the DOE Center of Excellence for the Synthesis and Processing of Advanced Materials as described in Sec. IV.

We were recently notified by DMS that our FY93 New Initiative proposal entitled "The Synthesis and Processing of Nano-Size Clusters for Energy Applications" has been approved for an FY94 start. The initial funding for this activity $(\$ 250 \mathrm{~K})$ is made up from some redirection of funds from other elements of our program plus some new funding from DMS. The goal of this new activity is to exploit our patented cluster synthesis technique to produce novel clusters with potential applications in energy technologies. Early emphasis will be on inexpensive clusters (e.g., Fe, $\mathrm{FeS}_{2}, \mathrm{MoS}_{2}, \mathrm{Ni}, \ldots$ ) as alternatives to precious metal catalysts for coal liquefaction and gassification, pollution control and solar photolysis. We feel that this work has the potential for major impact to many DOE programs.

Our interdisciplinary program utilizes a broad array of sophisticated, state-of-the-art experimental capabilities provided by other programs. The major capabilities include several molecular beam epitaxy and chemical vapor deposition facilities, a broad range of materials synthesis and 
processing facilities, clean rooms, ion-beam accelerators, laser-based diagnostics, advanced optical and surface spectoscopies, unique combined high-pressure/low-temperature/highmagnetic-field facilities, and electron, scanning tunneling and atomic force microscopies.

\section{The DOE Center of Excellence For The Synthesis and Processing of Advanced Materials. (S\&P Center)}

In addition to being involved in the technical program of the S\&P Center, Sandia is responsible for the overall coordination of Center activities. Summaries of our coordination efforts and the Sandia activities in the Center's program plan are given in Sec. IV, p. 10.

\section{Industrial Interacrions/Technology Transfer}

Our DMS-funded program has been responsible fully or in part for the generation of over 30 cooperative research and development agreements (CRADAs) with industrial partners. We also have several less formal collaborations. A summary is provided in Sec. V, p. 15.

\section{New Proposals}

This year we are submitting two proposals. Summaries can be found in Sec. VI.A., p. 20. The first is our New Initiative Proposal entitled "Weiting and Flow of Liquid Metals and Amorphous Ceramics at Solid Interfaces." It's purpose is to develop a new approach that will provide a scientific basis to understand the nanometer-scale structure, chemistry and flow properties of liquid metals and amorphous ceramics at solid interfaces. We will develop a fundamental understanding of the wetting and flow properties of interfacial liquids that combines:

1. new atomic scale methods for measuring the viscous response of liquids near well characterized interfaces,

2. theoretical simulations for liquid flow and stability, and

3. macroscopic wetting, spreading, and creep measurements that can be used to relate the results of fundamental experiment and theory to practical materials response.

The science involved is vital to many technological areas including soldering, brazing, welding, metal-matrix composites and the high temperature deformation of polycrystalline ceramics.

The second proposal is a re-submittal of the proposal we submitted last year. It is intended to be an enhancement to our Ceramics project and is ir response to the Metallurgy and Ceramics Branch's interest in ceramic toughening. It is entitled "Ceramic Toughening by Crack-Interface Bridging". Crack-interface bridging by grains is a potentially effective toughening mechanism; however, necessary quantitative characterization of the effects of grains boundaries on bridging is wanting. By combining analytical electron microscopy, microstructural control, and computeraided modeling, we propose to ascertain the effects of grain boundary structure on crack propagation and bridging, and to use this information to develop strategies for producing tougher ceramics. We will characterize both grain boundary chemistry and the distribution of relative crystallographic misorientations (texture) in single-phase polycrystalline ceramics using analytical electron microscopy and electron backscattered Kikuchi patterns. The effects of 
changes in the grain boundary character on the mechanical behavior will be measured using Vickers diamond indentations, bicrystals, fracture surfaces, and fracture toughness tests. The information will be used to fabricate fine-grained single-phase polycrystalline ceramics with enhanced toughness.

\section{Technical Program Highlights}

The technical accomplishments of the Program during the past year are summarized in Sec. VI.B. Highlights of the work include:

- Discovery of an ambient temperature/pressure process to prepare aerogels, which are exceedingly low density porous solids that have the highest insulating capacity of any material. (pp. 27 and 39)

- Fabrication of a new class of semiconductors comprising fractally-sequenced quantum wells and demonstration of highly efficient, broad-band emission from them. (pp. 28 and 48)

- Development of improved precursors and near equilibrium processing method for Tlbased high temperature superconducting films. (p. 50)

- Discovery of a dynamical quantum-mechanical mechanism through which large bipolarons attract leading to coalescence into a liquid (the precursor to bipolaronic superconductivity). (pp. 29 and 54)

- Discovery that He ion implantation-produced nanocavities in Si provide a new and more effective approach to impurity gettering. (pp. 30 and 44)

- New insights into adhesive bond formation and the nanomechanical properties of grains in polycrystalline films and of monolayer lubricants were obtained using the extraordinary force and displacement control of the Sandia Interfacial Force Microscope. (pp. 31 and 42)

- First direct observation of how small concentrations of dopants affect the surface chemistry governing CVD processes. (pp. 32 and 56)

- Quantitative determination of the energetics of trapped impurities on surfaces confirming the suspected role of impurities in influencing the growth of single crystals and epitaxial films. (pp. 36 and 52)

- Development of a comprehensive model to quantitatively predict ion-induced surface morphology evolution during low-energy ion sputtering. (pp. 35 and 46).

- Growth of the first large $\left(1 \mathrm{~cm}^{3}\right)$ single crystals of the refractory, wide-bandgap semiconductor $\mathrm{B}_{12} \mathrm{P}_{2}$ by a top-seeded solution method). (p. 54)

- Growth of ultra-high strength oxide dispersion-hardened Al films by a scalable pulsedlaser deposition technique. (p. 44) 


\section{Program Reviews}

Several elements of our DMS-funded program were reviewed by OPA panels during the past year and received high marks. The work in the Ceramics program dealing with solution-derived ceramics and the structure and connectivity of aerogels and polymers was judged to be an

"extremely strong project. The work completed to date has had a defining influence on the study of weakly-connected sol-gel systems. This project deserves continued support at a very high priority level."

Our Atomic Level Science of Interfacial Adhesion project was said to be

"a strong project deserving continuing priority support. It addresses issues of scientific importance that are in an area of interest to DOE. The combination of theory and experiment, particularly given the innovative experimental approach, make it likely that important new results in the field will be forthcoming."

Our project on Tailored Surfaces and Interfaces for Materials Applications was judged to be

"a very strong project. The research topic is interesting and important. The researchers are exceptionally equipped to make progress. The overall scope of the project is large, perhaps a little too large for the existing manpower."

The panel recommended tightening the focus of this project, and we are responding.

A review of all of Sandia's BES programs by the Basic Energy Sciences Advisory Committee (BESC) will take place on February 25, 1994. We are preparing for this review.

\section{Program Changes}

The titles of two of our projects have been modified to reflect a more accurate description of their current and planned future thrusts. The changes, which were conveyed to Alan Dragoo at DMS, are as follows: The "Advanced Growth Techniques for Improved Semiconductor Structures" project has been renamed "Advanced Growth Techniques and Science of Epitaxy." The "Strained-Layer Semiconductor Materials Science" project has been renamed "ArtificiallyStructured Semiconductors." This latter change also represents a closer tie to Focus Area I of the S\&P Center.

The Chemical Interfaces theme has been dropped from the Ceramics project largely because the PI, C.H.F. Peden, left Sandia, and the funding for the project is also tight. Additionally, due to difficulties in the interpretation of $\mathrm{Pb}$ NMR, the NMR activity in Ceramics has been redirected to Sn-containing precursors. We expect Sn NMR to provide the needed information for understanding the oligomeric species involved in complex ceramic film formation. 
Staff changes in the Program are as follows:

\section{Ceramics:}

CVD Sciences:

Novel Superconductors:

Adhesion:

Tailored Surfaces:

Borides:

\section{Additions}

W.G. Moffat

T.J. Boyle (post doc)

H. Moffat

N. Missert

C. Dirubio (post doc)

A. Wright

R. Stumph (post doc)

P. Yang (post doc)
Losses

C.H.F. Peden

C. Phifer

B. Dodson

A.C. Switendick

\section{Program Quality/Recognition}

1993 was another excellent year in terms of the significant accomplishments and productivity of our DMS program (as detailed in Sections IV.B and V.B of this Executive Summaries booklet) and in terms of the professional recognition received by our staff, both of which reflect on the quality of our BES program.

Richard Brow, a PI in our Physics and Chemistry of Ceramics program received the Schartzwalder Ceramics Prize. We received two DMS awards: 1. Sustained Outstanding Research in Metallurgy and Ceramics for our work on "Strained-Layer and Artificially Structured Materials"; and 2. Significant Implications for DOE Technologies in Metallurgy and Ceramics for our work on a "Interfaced Force Microscope."

Other program highlights include:

- Tom Picraux served as President of MRS during 1993.

- George Samara served as Co-Chair of Conference and Program Chair of the Joint 1993 AIRAPT/APS Conference on High Pressure Science and Technology. BESfunded work was prominent in the program.

We also organized symposia, chaired sessions, and presented numerous invited papers at national and international conferences (see individual program summaries for details). 


\section{The DOE Center of Excellence For The Synthesis And Processing of Advanced Materials}

This Center is a coordinated, cooperative, venture among the following laboratories: Ames Laboratory (Ames), Argonne National Laboratory (ANL), Brookhaven National Laboratory (BNL), Idaho National Engineering Laboratory (INEL), University of Illinois Materials Research Laboratory (UI/MRL), Lawrence Berkeley Laboratory (LBL), Lawrence Livermore National Laboratory (LLNL), Los Alamos National Laboratory (LANL), National Renewable Energy Laboratory (NREL), Oak Ridge National Laboratory (ORNL), Pacific Northwest Laboratory (PNL), and Sandia National Laboratories (SNL).

Its overall objective is "To enhance the science and engineering of materials synthesis and processing in order to meet the programmatic needs of the Department of Energy and to facilitate the technological exploitation of materials".

The Center's technical activities encompass many of the synthesis and processing (S\&P) elements of DMS's core programs at the laboratories. These technical activities have been organized under five Focus Areas each having a number of common themes. The focus areas and their coordinators are:

\begin{tabular}{lll}
\hline \multicolumn{2}{l}{ Focus Areas } & Coordinator(s) \\
\hline I. & Atomically-Structured Materials & $\begin{array}{l}\text { Daniel Chemla \& } \\
\text { Mark Alper (LBL) }\end{array}$ \\
II. & Polymers & Dale Schaefer (SNL) \\
III. & Ceramics & Linda Horton \& \\
IV. & Metals and Alloys & Jim Roberto (ORNL) \\
V. & Emerging Materials and Processes & Bob Dunlap (ANL) \\
& & Bruce Thompson (Ames) \\
& Overall Coordinator & George Samara (SNL)
\end{tabular}

Sandia has incorporated all the S\&P elements of its DMS-funded programs into the Center. In addition we have the responsibility for the overall coordination of Center programs.

\section{A. Coordination Meetings}

1. Germantown, May 6, 1993

The Center's Focus Area Coordinators and G. Samara met with Iran Thomas and other members of the staff for updates on the Center and the DMS program. A major objective of the meeting was to discuss and plan interactions with the Center's Industrial Steering 
Group (ISG). An agenda and plans for the first meeting and briefing for the ISG were developed.

2. Boston, November 30, 1993

This meeting was held in conjunction with the MRS Fall meeting and was attended by all laboratory coordinators and many members of DMS' staff. The ISG's observations and feedback received subsequent to the Center's meeting with them on July 19, 1993, were reviewed. Much of this meeting, however, was devoted to a discussion of and ideas for implementation of DMS' \$1-2M FY94 enhancement of Center activities

3. Albuquerque, January $12-13,1994$

This meeting was attended by the Focus Area Coordinators and G. Samara. The main agenda item was to identify and develop several coordinated, well-focused multilaboratory Center projects and to recommend them to DMS and the Laboratories. A few of these projects will be recommended for the $\$ 1-2 \mathrm{M}$ enhanced funding of Center activities. Seven projects were tentatively identified. These are: (1) high-rate metal forming, (2) material joining, (3) optimization of magnetic properties, (4) in-situ generated polymer composites, (5) Nano-structured materials for electronic and energy applications, (6) high-temperature corrosion coatings, and (7) processing for surface hardness. The scope and focus of each project will be refined based on inputs from the Laboratories and DMS, and the Center coordinators are expected to make their recommendations to DMS by the end of February 1994.

\section{B. The Center's Industrial Steering Group}

During FY93 we established an Industrial Steering Group (ISG) for the Center and defined its role. We also organized the first meeting with ISG which was held at LBL on July 18-19, 1993, and attended by representatives from all the labs. Presentations about the Center were given by Iran Thomas, George Samara and the Focus Area Coordinators, and the agenda allowed for considerable discussion with members of the ISG.

\section{ISG Membership}

The members of the ISG are

\begin{tabular}{lll}
\hline \multicolumn{2}{l}{ Focus Areas } & Group Member(s) \\
\hline I. & Atomically-Structured Materials & John Carruthers (Intel) \\
II. & Polymers & Tom Clarke (IBM-Almaden) \\
III. & Ceramics & Uma Chowdhry (Dupont) \& \\
& & Dave Johnson (AT\&T Bell Labs) \\
IV. & Metals and Alloys & Neil Paton (Howmet) \\
V. & Emerging Materials and Processes & Tom Anthony (GE) \\
& & Hylan Lyon (Marlow Industries)
\end{tabular}


Regretfully, John Carruthers resigned late last fall because he was given a new and different assignment at Intel. We have several candidates for his replacement; however, at the Albuquerque Coordination Meeting (January 12-13, 1994) it was decided that selection of a replacement should await finalizing the new Center projects.

\section{Role of the ISG}

The role we defined for, and discussed with, the ISG is to:

- Become familiar with the Center's technical activities and comment on their value to industry.

- Provide information from an industrial perspective--insight into and vision of what is important. Identify technologies and needs that will be important in the next 5 years, 10 years, etc. ...Identify technological barriers.

- Influence (steer) the direction of the program. Help develop ideas which can make the Center more effective.

- Take home information and foster closer interactions between the Center and industry. Help the Center develop more effective mechanisms for working with industry.

- Argue for and support the Center's programs.

\section{ISG Observations/Feedback}

A summary report of the July 18-19 meeting was prepared and distributed to DMS and the labs. Among the general observations made by the ISG are the following:

- The ISG feels that National Laboratories should focus on those things that universities cannot do because they

- are too expensive for universities (e.g., the new SNL/IBM fab line)

- require major facilities

- require interdisciplinary teams

- ISG suggested closer coupling of research to advanced manufacturing.

"Every problem in manufacturing today is a materials problem". We (in U.S.) have not well coupled fundamental research and manufacturing. This interface starts with materials science. National Laboratories are the logical place to emphasize this interface, but there needs to be balance between the tie to manufacturing and the more fundamental tech base.

- There are strong industrial needs in the areas of phase equilibria, stability and nonequilibrium phases.

- Small companies do not have a lot of research input. They do not know how to tap national laboratories.

- In small companies, people who do manufacturing do the "research". 
- National Laboratories should help small companies who are the ones creating the jobs. Those companies do not have many resources, or many options. They have to deliver or go down the tube quickly.

- National laboratories have an important role in view of declining industrial research.

- Materials characterization capabilities of the laboratories are superb.

- You learn about materials processing during process integration.

ISG members caucused after being briefed about the Center and its technical activities and provided feedback. The following are some of the main observations made:

- The technical activities of the Center are of great interest; the scope is impressive. The National Laboratories bring to the table great strength in materials and characterizations not available elsewhere in the Nation.

- What you are trying to do with the Center is not easy, but worth trying.

- Center is not a "Center", but a loose affiliation of projects that exist for their own reasons.

- Focus is missing in projects. They are too diverse. Focus should not be on materials for their own sake; better to focus on processes.

- In a true center, goals and priorities are established by give and take. How do projects meet national needs should be the basis for rational decisions.

- Many materials scientists have a proclivity for working in their sand box. Materials scientists do their best work when challenged by applications (e.g., advances in fiber optic technology).

- Other vehicles (than CRADAs) for collaboration with industry need to be identified. CRADAs take too long; are too legalistic.

- Reexamine the motivation (to the staff) for enhancing the interaction between laboratories and industry.

- The Semiconductor Industry has a well-developed road map which defines the important materials research issues. Other industries need similar road maps and statements of challenges.

Following the meeting, ISG members were requested to provide their views on two issues: (1) How can the Center establish more effective alliances with industry, and (2) Ways to make the Center more effective and valuable. The responses received can be summarized as follows. On the first issue the recommendations are:

- Joint projects including exchange of people

- Participation in industry road maps development

- Make CRADAs easier and faster 
On the second issue the recommendations are:

- Focus on a few projects and execute them well

- Need a strategy and decision-making process which involve all stakeholders

- Publicize the Center; Newsletters are common, but "we seldom have time to read them all" 


\section{Industrial Interactions and Technology Transfer}

\section{A. CRADAs and Contracts}

The research results and capabilities of our DMS-sponsored program have been responsible fully or in part for the generation of over 30 cooperative research agreements or contracts with industrial partners. Details are given in the Table on the following pages.

While all projects in our Program are involved in industrial interactions and technology transfer, two projects, CVD Sciences and Physics and Chemistry of Ceramics, stand out in being involved in over half of the industrial agreements. The spin-off from our DMS-funded CVD project have included the design, construction and delivery of a scaled-up rotating disk CVD reactor to SEMATECH, the development of detailed chemical models for silicon deposition from silane in another SEMATECH CRADA, and for silicon nitride deposition processes for composite materials in a DARPA-funded project in advanced manufacturing with United Technologies Research Center (UTRC). In the DARPA/UTRC project an experimentally supported computer model simulating the complex fluid mechanics and chemical reactions of silicon nitride deposition was developed and used to design a scaled up commercial reactor. Current DARPAsponsored projects include work with the Norton Company on thick-film diamond synthesis for electronics packaging and the 3M Company on fiber synthesis for metal-matrix composites. Ongoing projects in SEMATECH CRADAs include work on the design and control of LPCVD diffusion furnaces.

The CVD project has also led to several DOE/DP Technology Transfer Initiative programs: for example the InfraRed Imaging Spectroscopy (IRIS) technique is being applied to measurement of critical kinetic rates in plasma processes in a CRADA program with Texas Instruments. Additional CRADAs are underway with Hewlett-Packard, DuPont, Motorola, and Emcore, and we have numerous additional inquiries from industry, including Intel, Spire and Ford Glass Division. In the latter case, Ford is interested in teaming with Sandia to develop atmospheric pressure CVD (APCVD) processes for depositing solar control optical coatings on plate glass, an application that would have significant impact to energy conversation for this country. We are investigating the possibilities of initiating a CRADA effort funded from our DMS CVD Science project to share our expertise in this area with Ford in return for learning more from Ford about the chemical systems and processes that are applicable to optical coatings.

In the Ceramics project our research on ferroelectric thin films has led to two CRADAs with Radiant Technologies on nonvolatile memories and to our involvement with the ARPA-sponsored NCAICM initiative, also on nonvolatile memories. Superconductor Components, Inc. has obtained a license to market high quality superconductor powders produced by our patented solution process, and we continue to consult with this company. Our sol-gel films research has led to our involvement in three CRADAs and a contract with the Gas Research Institutue to develop porous materials for methane storage. Two fo the CRADAs are ARPA-funded, one with $3 \mathrm{M}$ on fiber reinforcement and the other with a 7-member industrial consortium on nonlithographic processing. The third CRADA is with AMOCO and deals with the development of 


\begin{tabular}{|c|c|c|c|}
\hline Company & Agreement & Project & DMS-Funded Progenitor(s) \\
\hline Radiant Technologies & DP/TTI-CRADA & Nondestructive Readout Nonvolatile Memories: (Semiconductor/Ferroelectric) & Ferroelectric Ceramics \\
\hline Radiant Technologies & DP/TTI-CRADA & Optical Read-Write Memory & Ferroelectric Ceramics \\
\hline SEMATECH & DP/TTI-CRADA & Benchmarking Microelectronics and Manufacturing Equipment & CVD Sciences \\
\hline SEMATECH & DP/TTI-CRADA & $\begin{array}{l}\text { Microelectronics Manufacturing Technology-Semiconductor Equipment Reliability } \\
\text { Modeling and Design. }\end{array}$ & $\begin{array}{l}\text { CVD Sciences; Strained Layer } \\
\text { Semic } \text {;nductors }\end{array}$ \\
\hline SEMATECH & DP/TTI-CRADA & Contamination-Free Semiconductor Manufacturing & Energetic Part. Synthesis \\
\hline Hewlett Packard & DP/TTI-CRADA & Advanced Manufacturing Techniques for Optoelectronics & $\begin{array}{l}\text { Strained-Layer } \\
\text { Semiconductors; Ceramics }\end{array}$ \\
\hline Hewlett Packard & DP/TTI-CRADA & Electronic, Optical and Thermodynamic Properties of Visible Light Emitting Diodes & $\begin{array}{l}\text { Strained-Layer } \\
\text { Semiconductors }\end{array}$ \\
\hline Texas Instruments & DP/TTI-CRADA & Measurements of Kinetic Rates in Plasma Processing. & CVD Sciences \\
\hline DuPont & DP/TTI-CRADA & Diamond Film Synthesis and Processing. & CVD Sciences \\
\hline $\begin{array}{l}\text { Digital Instruments, } \\
\text { AT\&T, Univ. of NM }\end{array}$ & DP/TTI-CRADA & Non-Contact Atomic Level Interface Force Microscope. & Interfacial Adhesion \\
\hline Conductus, Inc. & DP/TTI-CRADA & Confocal Resonator Imaging System for Surface Analysis & Novel Superconductors \\
\hline Cray Research & DP/TTI-CRADA & Massively Parallel Electronic Structure Methods for CRAY's MPP Supercomputer & $\begin{array}{l}\text { Strained-Layer } \\
\text { Semiconductors }\end{array}$ \\
\hline $\begin{array}{l}\text { Charles Evans \& } \\
\text { Assoc. }\end{array}$ & $\begin{array}{l}\text { BES/MS- } \\
\text { CRADA }\end{array}$ & New Light-Element Depth Profiling Technique & Energetic Part. Synthesis \\
\hline Motorola & DP/TTI-CRADA & Advanced SiGeC Materials for High-Performance Digital Microelectronics & Adv. Growth \\
\hline NCAICM & ARPA-CRADA & Manufacturing Technology for 1 Megabyte Ferroelectric Nonvolatile Memory & Ferroelectric Ceramics \\
\hline 3M & $\begin{array}{l}\text { ARPA-Joint } \\
\text { Project }\end{array}$ & Drying of Fibers - File Reinforcement Project & Sol-Gel Films \\
\hline Amoco & IWRP-CRADA & Membranes for Commercial Separations & Sol-Gel Films \\
\hline $\begin{array}{l}\text { 7-Member Display } \\
\text { Consortium }\end{array}$ & ARPA-CRADA & Non-Lithographic Processing & Sol-Gel Films \\
\hline
\end{tabular}




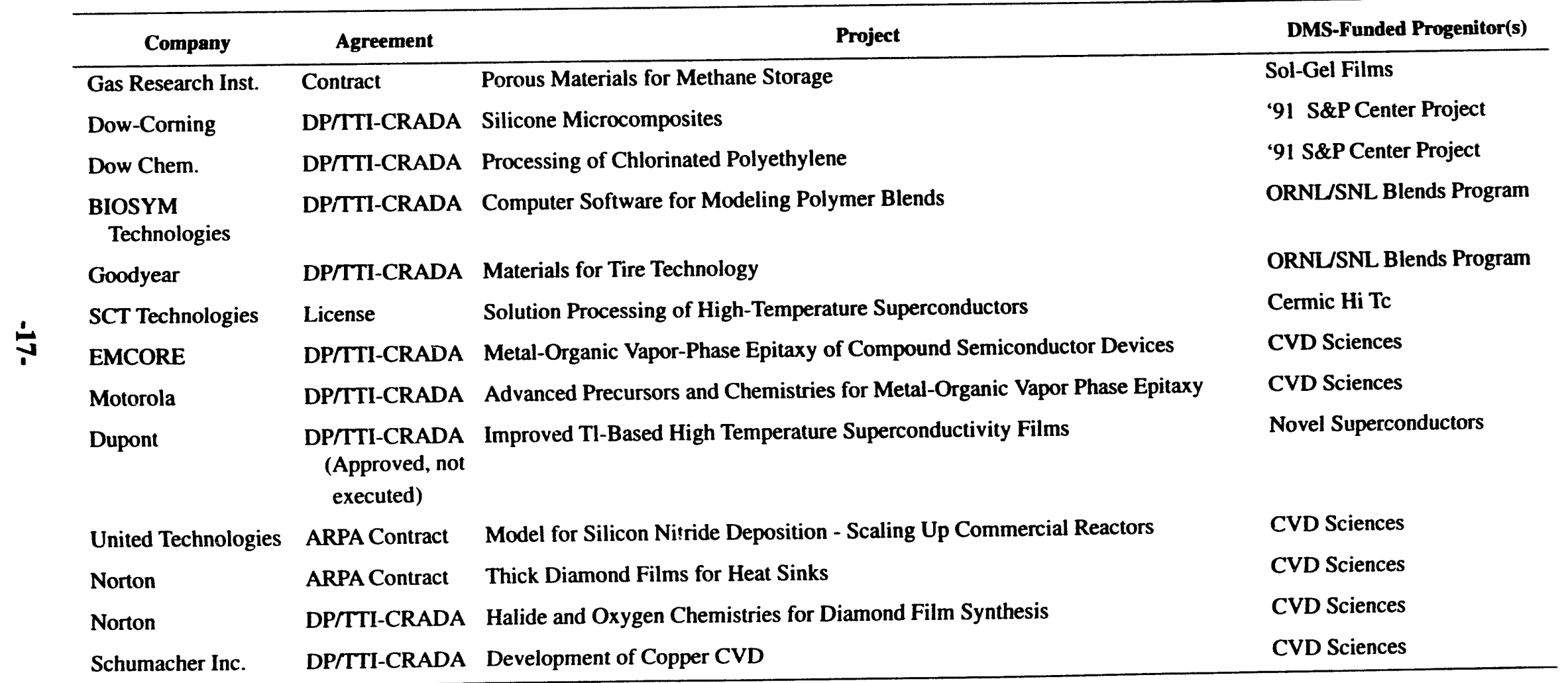


membranes for gaseous separations. Other spin-offs from the Ceramics project are listed in the Table.

All of the industrial connections summarized above and in the Table point clearly to the value and impact of our DMS-funded research. It is critical that we maintain the focus of this research on the development and enhancement of new reserach tools and capabilities, for it is these tools that are the foundation of the applied programs.

\section{B. Other Industrial Interactions}

Our DMS-sponsored research has also been responsible for several other less formal, but significant, industrial interactions and/or technology transfer activities. These include the following:

1. Semiconductor Heterostructure Mirrors for Vertical Caviv Surface-Emitting Lasers - The study of semiconductor heterostructure mirrors, leading to high Q Fabry-Perot laser cavities now used by industry (Bandgap Technology, Photonics Research), was initiated in our program on strained-layer semiconductors. The synthesis of these structures and the study of their physical properties was funded largely by DMS. The technology was recently transferred to Bandgap Technology Corporation which markets $860 \mathrm{~nm}$ lasers for optical communications and imaging applications.

2. Gettering of Impurities - Within the Energetic-Particle Synthesis and Science of Materials project it was discovered that microscopic cavities within a silicon wafer trap (more effectively than with other means), metal impurities that would otherwise be highly detrimental to electronic devices on the wafer. These cavities can be formed by ionimplanting $\mathrm{He}$ and then heating the material to $700^{\circ} \mathrm{C}$ or above. After this discovery was released in 1993, expressions of interest and requests for additional information were received from SEMATECH and twelve semiconductor companies, one of whom has proposed to initiate a CRADA.

3. Surface Treatments - Within the Energetic Particle Synthesis and Science of Materials project it was discovered that ion implantation of oxygen into aluminum can produce an extremely high density of nanometer-size aluminum-oxide particles, and that the resulting alloy has high mechanical strength (in the gigapascal range typical of high-strength steels), and substantially reduced friction and wear during sliding contact. Release of this discovery in trade journals led to requests for information from 41 companies, and at least two of these are known to be exploring technological applications.

Previously (more than 5years ago) we discovered that the implantation of titanium and oxygen into ferrous alloys creates an amorphous layer with greatly reduced friction $(x 1 / 2)$ and increased wear $(x 10)$ resistance. This surface treatment process was transferred to industry, and, for example, is still used by Spire Corporation for surface treatment of hard bearing steels to achieve improved tribological properties. 
4. Dodson-Tsao Model of Strained-Layer Stability and Metastability - This work provided a unified approach to understanding the stability limits of strained layer films and the kinetics of strain relaxation. It was crucial in the quantitative understanding of strain relaxation and stability in semiconductor structures. These types of structures are now used commonly in the edge-emitting diode lasers found in CD-ROM players and ultrafast pseudomorphic HEMT devices used in microwave applications.

5. Stress Corrosion of Ceramics - Our earlier DMS-funded work in this area led to an AT\&T:funded project to apply fundamental models for glass fracture and glass surface reactivity to develop optical fiber cables with improved reliability.

6. Consortium for Advanced Synthesis and Processing of Electronic Materials - A consortium of 5 companies and 4 universities, led by Texas Instruments and Hughes Aircraft and funded by ARPA, has been formed to study ways to advance the synthesis and processing of new electronic materials. The major goal of this project is to enhance molecular beam epitaxy (MBE) technology to improve U.S. competitiveness in semiconductor structures. Sandia has been chosen as an associate member of this team. Reflection mass spectrometry (REMS), developed under the DMS Advanced Growth project, has been targeted for development as an on-line diagnostic for monitoring of surface reactivity during III-V quantum well synthesis.

7. SEMATECH Task Force on Surface Microroughness - SEMATECH has recognized the importance of monitoring and control of nanoscale surface roughness during semiconductor wafer production for next generation electronic devices. A task force has been formed to close the technology gap in particle counting and microroughness determination. SEMATECH has enlisted our help as a designated resource on surface roughness to help in the development and calibration of various techniques for determining surface roughness. We have used the EDXRR (energy-dispersive X-ray reflectivity) technique developed in the DMS Advanced Growth project to measure the surface roughness of as-prepared wafers.

8. Boron-Rich Solids for Energy and Radiation Applications - We have consulted extensively with two companies who have expressed strong interest in our DMS-funded work on boron-rich solids. One of the companies, High $\mathrm{Z}$, is interested in the thermoelectric properties of these materials and already markets a product, and the other, Science and Engineering Associates (SEA) is interested in energy conversion devices and in the extreme radiation tolerance of borides.

9. Surface Science Codes - Discussions have been initiated with Biosym Corp., to develop commercial software based on computational methods developed in our DMS-funded Tailored Surfaces project. 


\section{Research Program Summaries}

\section{A. New Proposals}

1. New Initiative Proposal

Wetting and Flow of Liquid Metals and

Amorphous Ceramics at Solid Interfaces $\ldots \ldots \ldots \ldots \ldots \ldots \ldots$

2. Other Proposal

Ceramic Toughening by Crack-Interface Bridging $\ldots \ldots \ldots \ldots 24$ 


\section{New Initiative Proposal}

\section{Wetting and Flow of Liquid Metal and Amorphous Ceramics at Solid Interfaces}

\section{Principal Investigators}

T. A. Michalske, N. D. Shinn, F. G. Yost - SNL

R. Cannon, R. O. Ritchie - LBL

University and Industrial Collaborators

U. Landman - Georgia Tech

J. Mann - Case Western University

D. Marshal - Rockwell International

W. Winterbottom - Ford

\section{Objectives}

The properties of thin liquid metal and amorphous ceramic layers play an important role in the synthesis, processing and stability of materials that are being developed to meet the current and future needs of energy and many other technologies. Specific examples include:

- Metal and ceramic joining technologies such as soldering, brazing and welding depend on the ability of a liquid phase to wet the interface between two solids.

- The fabrication of light-weight metal matrix structural composites involves the infiltration of a liquid metal matrix into a porous ceramic precursor.

- The high temperature deformation of polycrystalline ceramics is controlled by viscous flow in the amorphous grain boundary phase.

Microscopic flow processes are clearly important to the stability of atomically thin grain boundary films. Similarly, the spread of a macroscopic liquid is controlled by atomic-level motion at the leading edge of the droplet. Our lack of microscopic understanding limits the ability of current fluid flow models to properly predict wetting and flow of liquids at solid interfaces. As a result of this limitation we are unable to anticipate the formation of microstructural defects that can seriously limit the performance of materials in critical components. Clearly, great technological and economic gains can be realized by placing the field of interfacial liquid flow and stability on a firm scientific basis which will allow the selection of materials combinations and processing conditions for defect-free manufacturing.

The purpose of this new initiative is to develop a new approach that will provide a scientific basis to understand the nanometer-scale structure, chemistry and flow properties of liquid metals and amorphous ceramics at solid interfaces. We will develop a fundamental understanding of the wetting and flow properties of interfacial liquids that combines:

1. new atomic scale methods for measuring the wetting and flow of liquids near well characterized interfaces, 
2. theoretical simulations for liquid flow and stability, and

3. macroscopic wetting, spreading, and creep measurements that can be used to relate the results of fundamental experiment and theory to practical materials response.

Key to our unique approach is the ability to make detailed measurements of the wetting and flow of atomically thin, well characterized liquid metal and amorphous ceramic interfacial layers. These measurements will provide a common point that will permit interactions extending from atomic-level theory to practical wetting and flow measurements and will provide fundamental insight into the factors controlling the wetting and flow of thin liquid layers.

\section{New Experimental Techniques Will Provide Microscopic Understanding}

Presently, efforts to develop and veriify scientific models for wetting and flow at liquid/solid interfaces are restricted by the lack of quantitative experimental results for the structure and viscous response of well-characterized fluid layers. The macroscopic nature of contact angle, bulk viscosity and creep measurements cannot supply the information necessary to develop and verify microscopic models.

The time is ripe to apply new experimental capabilities that can be used to examine the structure and flow properties of well characterized liquid/solid interfaces. We will develop a new approach that is based on acoustic wave (AW) techniques and thin film deposition and analysis to directly measure the viscoelastic properties of thin liquid metal and amorphous ceramic layers. The detailed measurements derived from this approach will permit direct comparison with results from theoretical calculations and are key to our ability to develop and verify fundamental models for the properties of liquid/solid interfaces.

Although acoustic wave devices are generally known for their use in measuring the thickness of adsorbed layers, recent studies have demonstrated that they are also sensitive to the viscoelastic properties of liquid films as thin as one monolayer. In addition, new optical and $\mathrm{x}$-ray techniques have been developed which allow us to fully characterize the buried liquid/solid interface in metal and ceramic systems. We will take advantage of these new capabilities by depositing well characterized metal and amorphous ceramic layers on acoustic wave devices and simultaneously monitoring their viscoelastic and structural evolution as they are heated to form liquid/solid interfaces.

\section{Program Plan}

We will begin by examining atomically sharp liquid/solid interfacial structures, move on to chemically reactive and atomically diffuse interfaces, including their temporal evolution, and then use the microscopic results to derive new models to predict complex macroscopic spreading and creep behavior. This systematic progression through increasingly complex but well-defined interfacial structures will enable us to evaluate the role of interface morphology, impurities, surfactants, and chemical reactions in determining macroscopically observed wetting, spreading, and interfacial fluid flow.

We will begin our atomic level studies on metal/metal interfaces in order to take advantage of our extensive experimental modeling experience with these systems. We will extend our approach to amorphous ceramic liquid layers. Of particular interest are thin silicate layers that are commonly 
found in polycrystalline ceramic grain boundaries. By controlling the composition of the silicate layer, we can examine the role of additives on the wetting and flow properties of the thin boundary layers.

\section{Multi-disciplinary Research Team}

The research plan calls for a multi-institutional, multi-disciplinary effort involving SNL, LBL, Georgia Tech, Case-Western University, Rockwell, and Ford. This project builds upon strengths of the laboratories involved, yet represents decidedly new directions for each. SNL has a high degree of expertise in the atomic scale, and macroscopic properties of metallic systems. The soldering center at SNL has extensive ties to industrial interests in liquid metal processing problems. LBL will contribute its expertise in the processing and properties of high temperature ceramic systems. Uzi Landman at Georgia Tech has developed world-class dapabilities/for atomic-scale modeling of bi-metal interfaces, and J. Mann at Case Western University will contribute his expertise in the application of thermodynamic modeling of liquid interfaces. Rockwell and Ford are directly involved in joining and high temperature ceramic materials processing and properties.

\section{Budget}

We request $\$ 650 \mathrm{~K}$ per year for a minimum of 3 years. Approximately $15 \%$ of this funding will be allocated to our university collaborators. 


\title{
Other Proposal
}

\section{Ceramic Toughening by Crack-Interface Bridging}

\author{
Principal Investigators \\ Jill Glass, Ed Beauchamp, Elizabeth Holm, and Joe Michael \\ Goals
}

To establish the relationship between grain boundary structure and chemistry, and crack propagation in single-phase polycrystalline ceramics (SPPC). Achieving this goal will provide the information necessary to develop optimal toughening strategies.

\section{Background}

Improving the mechanical performance of ceramics has motivated a substantial portion of ceramics research for decades. Although a variety of toughening schemes has been demonstrated for special classes of materials, the optimization of fine-grained, single-phase isotropic ceramics is yet to be realized.

Polycrystalline ceramics can exhibit increased crack resistance with increasing crack length ("rising R-curve behavior"). This desirable response arises because toughening mechanisms are activated in the wake of the crack tip, such as fiber or grain bridging. We know that R-curve behavior is promoted by grain bridges, but little is known about bridge formation. This knowledge gap is due to the paucity of quantitative information on the effects of the relative crystallographic misorientations (RCM's) between adjoining grains and grain boundary chemistry on crack propagation and grain bridging.

We believe that optimal toughening is achieved when the fracture toughness of the grain boundary is only slightly lower than that of the grain interior, and there is a distribution of grain boundary energies permitting intergranular and transgranular fracture. Achieving even this simple goal requires detailed knowledge of the factors controlling grain boundary strength. We need to determine how the RCM between adjoining grains affects the strength of the grain boundary, as well as the effects of RCM on impurity segregation, the other major factor controlling grain boundary strength.

The evolution of boundaries during grain growth and changes in the distribution of RCM (less random) appear to play an important role $n$ enhancing bridging behavior because $\mathrm{R}$-curve behavior effects are strongest at larger grain sizes. Changes in the relative amounts of intergranular and transgranular fracture as grain size increases, which indicate a change in the strength of the boundaries, support this supposition. Unfortunately the grain in toughness at larger grain sizes is offset by a loss of strength. It would be a boon to achieve strong R-curve behavior at fine grain sizes by producing grain boundaries and distributions of RCM reminiscent of those that promote bridging behavior in the larger grained ceramics. To achieve this goal, changes in impurity segregation, and changes in the distribution of RCM during grain growth must be identified. 


\section{Approach}

- Materials Preparation: Magnesium aluminate spinel $\left(\mathrm{MgAl}_{2} \mathrm{O}_{4}\right)$ is an ideal system to begin our study. Spinel has a cubic crystal structure which simplifies the identification of the distribution of RCM's and eliminates anisotropy as a factor influencing crack propagation and bridge formation. We will use commercially prepared $\mathrm{MgAl}_{2} \mathrm{O}_{4}$ with a range of grain sizes as a startling point of establishing the role of RCM's on crack propagation and R-curve behavior. We will use materials prepared with and without $\mathrm{LiF}$, a common sintering aid, to establish the role of impurity segregation on the distribution of RCM's and crack propagation.

As the program develops, we will produce ceramics with controlled grain boundary chemistry and distributions of RCM by exploiting powder preparation technology developed through our BES program on the Chemistry and Physics of Ceramics. Specifically Jim Voigt specializes in the production of ceramic powders with tailored composition, particle size and distribution, and morphology.

- Analytical Methods: The distribution of RCM as a function of grain size and impurity level will be measured using electron back-scattered Kikuchi patterns in the scanning electron microscope. Grain boundary structure and chemistry will be measured using analytical electron microscopy (AEM), which combines scanning transmission electron microscopy with electron and $\mathrm{x}$-ray detectors to collect the signals generated when the electron beam interacts with a thin specimen.

We will take advantage of recent advances in AEM that allow quantitative characterization of the distribution and level of impurities in the vicinity of and on grain boundaries. Joe Michael received the 1991 Burton Medal from the Electron Microscopy Society of America for his work on spatial resolution effects in AEM.

- Fracture Analysis and Mechanics: We will determine how changes in the grain boundary character influence bridge formation and R-curve behavior. Working with well-characterized materials (RCM and chemistry) we will determine how these factors influence crack propagation (intergranular vs. transgranular) and bridge formation. Crack propagation can be characterized using fracture surfaces (bicrystals and polycrystals), which can also be used to provide bulk grain boundary chemistry using Auger electron microscopy. Recent advances in x-ray tomography may also make it possible to map crack propagation in three dimensions with a resolution smaller (approaching $2 \mu \mathrm{m}$ ) than that of grain sizes we wiii be utilizing. E. Beauchamp and J. Glass are experts in ceramic fracture.

- Modeling: Expanded computer capabilities make it possible to perform meaningful threedimensional simulations of grain growth and crack propagation that account for differences in the grain boundary energy. Elizabeth Holm is an expert in the simulation of microstructural evolution and other materials's related phenomena.

- Improving Toughness: We plan to enhance bridging and rising R0curve behavior by producing materials with the appropriate distributions of grain boundary strengths. Approaches include grain boundary doping and/or producing controlled distributions of RCM's using powders with tailored composition, particle size and distribution, and morphology.

Budget

$\$ 300 \mathrm{~K} /$ year. 


\section{B. Existing Programs}

\section{Significant Accomplishments}

1. Ambient Temperature/Pressure Processing of Aerogels ................27

2. Broadband Light Emission From Fractal Quantum Wells $\ldots \ldots \ldots \ldots \ldots \ldots .28$

3. Phonon-Mediated Attraction Between Large Bipolarons:

Formation of a Bipolaronic Liquid . . . . . . . . . . . . . . . . . . .29

4. New, Stronger Mechanism for Impurity Gettering in Silicon

Attracts Industry and Government Interest. .................. 30

5. Interfacial-Force Microscopy Probes the Mechanical Properties of Single, Nanometer-Size Metal Grains . . . . . . . . . . . . . . . . . 31

6. Direct Observation of Boron-Dopant Induced Effects on SiO2 CVD ........32

7. Band Structure Effects in Fullerene-Based Superconductors.............33

8. A New Understanding of Electron Cyclotron Resonance Plasmas Permits Controlled Hydrogen Treatment of Electronic Materials ..........34

9. Unified Model of Roughening Instability and Surface Evolution During Low Energy Ion Bombardment ................35

10. Direct Observation of Substitutional-Atom Trapping on a Metal Surface ......36

11. Infrared Spectroscopy of Adsorbates on GaAs Surfaces ................ 37 


\section{Ambient Temperature/Pressure Processing of Aerogels KC 020101}

Technical Accomplishment:

A BES-funded research team from Sandia National Laboratories and the University of New Mexico discovered an ambient temperature/pressure process to prepare aerogels, exceedingly low density porous solids that have the highest insulating capacity of any material. The new process eliminates the need for high temperatures and pressures previously required to remove the solvent from the wet precursor gel (so-called supercritical drying).

The conventional "supercritical" method of aerogel processing requires a high-pressure autoclave. The temperature and pressure are increased until the critical temperature and pressure of the pore liquid are exceeded. The supercritical pore fluid is then removed from the gel while maintaining the temperature above the critical temperature. The pore fluid is removed under supercritical conditions where there is no liquid-vapor interface and hence no capillary pressure that would collapse the gel structure.

In the new method, the capillary pressure is decreased toward zero by chemical modification of the surfaces. Not only do the dried gels prepared by the low temperature/pressure process have equivalent physical properties to conventional aerogels, but the pore surfaces are hydrophobic, avoiding condensation of water in the pores, which would otherwise serve to degrade aerogel properties.

\section{Significance:}

Aerogels are unique solids that are up to $99 \%$ air. Such high porosities confer a number of useful properties to aerogels including high surface areas (often exceeding $1000 \mathrm{~m} 2 / \mathrm{g}$ ), low refractive indices $(n<1.1)$, low thermal loss coefficients $(<0.5 \mathrm{~W} / \mathrm{m} 2 \mathrm{~K})$, and low sound velocities $(\leq 100$ $\mathrm{m} / \mathrm{s}$ ), leading to applications in catalysis, Cerenkov detectors, superinsulation, and acoustic impedance matching.

Because the new process is much less energy intensive than conventional aerogel processing, it is being considered by a number of industries for low cost production of thermal insulation What's more, the process is completely compatible with liquid-based coating operations. This aspect should allow the production of aerogel films with ultra-low dielectric constants, refractive indices, and thermal loss coefficients for applications in microelectronics, optics, displays, insulation, etc. Thin film production is not possible by conventional aerogel processing.

(Research performed by R. Deshpande and D. M. Smith of the University of New Mexico and C. J. Brinker of Sandia National Laboratories) 


\section{Broadband Light Emission From Fractal Quantum Wells KC 020105}

\section{Technical Achievement}

We have produced a new class of semiconductor materials comprising fractally sequenced quantum wells. The material was grown by metal-organic vapor phase epitaxy of thin $(\sim 100 \AA)$ layers of many different $\mathrm{Al}_{\mathrm{x}} \mathrm{Ga}_{1-\mathrm{x}} \mathrm{As}$ compositions. The composition is varied in a fractal sequence between layers to create a highly branched, self-similar distribution of quantum wells. The resulting material has hundreds of localized energy states for electrons and holes. The localization is controlled simply by changing the layer thicknesses and compositions. Such changes lead to dramatic changes in the electronic density of states, optical absorption strength, and electron-hole energy relaxation rates. Luminescence from these new materials is highly efficient and extends over a very broad, selectable range of wavelengths.

\section{Significance}

Illumination sources are vitally important to human society. Each day, about 10 kilowatt-hours per person are dissipated to illuminate homes, offices, shops, schools an vehicles. This adds to a total of to 2,500,000,000 kilowatt-hours dissipated in the United States alone! Only a small fraction of this power is useful. Most illumination sources are tungsten bulbs or fluorescent tubes. Tungsten bulbs produce $99.5 \%$ heat and $0.5 \%$ light, are inexpensive, but last less than 2 years. Fluorescent tubes are more efficient (few percent) but use phosphors which are toxic to humans and environmentally undesirable when disposed. Semiconductor materials can operate with 50 to 70 percent efficiency, last for decades, and can be disposed without harming the environment. Semiconductors which emit broadband light, as we have demonstrated, may have an important role to play in future illumination technology. We have a proposal under consideration by the Advanced Energy Projects Division of BES to develop fractal lattice and related artificially structured semiconductors as broadband light emitters.

(Research performed by P. L. Gourley, R. P. Schneider, Jr., C. P. Tigges, T. M. Brennan, B. E. Hammons, and A. E. McDonald, Sandia National Laboratories). 


\section{Phonon-Mediated Attraction Between Large Bipolarons: Formation of a Bipolaronic Liquid}

\section{KC 020205}

\section{Technical Achievement}

A dynamic quantum-mechanism through which large (multi-site) bipolarons attract one another has been discovered. In particular, as a result of their polarizabilities, large bipolarons comes about because the solid's zero-point vibrational energy falls as the separation between bipolarons decreases. The dynamic and quantum-mechanical natures of the attraction are reflected in the attractive interaction between bipolarons being proportional to a phonon energy, $h \omega$, where $h$ is Planck's constant and $\omega$ is the characteristic phonon frequency. Through this attraction large bipolarons can coalesce in to a liquid. The phase diagram for the bipolaronic liquid has been calculated. At finite temperatures a large-bipolaronic liquid can only form within restricted ranges of carrier densities.

\section{Significance}

Resistanceless flow in superconductivity and superfluidity is associated with an attractive interaction between carriers. For example, the attraction between ${ }^{4} \mathrm{He}$ atoms leads to their forming liquid. Below a critical temperature this Bose liquid becomes superfluid. A growing number of experiments find that the charge carriers in oxide superconductors manifest characteristics of bipolarons, charged bosons. The present work finds an interaction through which these bipolarons can coalesce into a charged Bose liquid, the precursor to bipolaronic superconductivity. Finding superconductivity only with restricted ranges of carrier densities, is consistent with having bipolaronic superconductivity in the high- $T_{c}$ superconductors.

(Performed by D. Emin, Sandia National Laboratories) 


\section{New, Stronger Mechanism for Impurity Gettering in Silicon Attracts Industry and Government Interest KC 020103}

Scientific Achievement:

Research over the past two years has demonstrated that microscopic cavities in Si are strong traps for two representative impurities from the highly detrimental transition-metal elements, namely $\mathrm{Cu}$ and $\mathrm{Ni}$. The cavities are formed by ion-implanting $\mathrm{He}$ and then annealing at temperatures around $700^{\circ} \mathrm{C}$, and the metal atoms are bound on the chemically reactive internal surfaces that result. A key point for the application of this effect to impurity control in $\mathrm{Si}$ wafers is that the binding of $\mathrm{Cu}$ and $\mathrm{Ni}$ impurities in cavities is substantially stronger than in precipitates of the respective metal-silicide phases, which is the basis for most current impurity gettering. In the case of $\mathrm{Cu}$ atoms, for example, the binding energy expressed relative to untrapped atoms in solution is $2.2 \pm 0.2 \mathrm{eV}$ for cavity walls and $1.7 \mathrm{eV}$ for silicide precipitation. This means not only that the residual concentration of ungettered metal atoms can be reduced to a value that is smaller by several orders of magnitude, but also that pre-existing precipitates of metal silicides in undesirable locations of the wafer can, for the first time, actually be dissolved.

\section{Significance:}

The requirements on metal-impurity control in $\mathrm{Si}$ wafers are becoming increasingly stringent as the dimensions of microelectronic devices shrink to well below one micrometer. Specifications projected by SEMATECH now extend to as low as $10^{8}$ atoms per $\mathrm{cm}^{2}$ of wafer surface. As a result, gettering methods that are widely and successfully used today can be expected to become progressively inadequate. Recognition of this fact is reflected by widespread interest in new approaches to gettering that offer potentially superior performance. Since the present discovery was released to trade journals in 1993, expressions of interest and requests for additional information have been received from SEMATECH and twelve semiconductor companies, one of whom has expressed a desire to establish a CRADA.

(Research performed by S. M. Myers, D. M. Follstaedt, and D. M. Bishop, Sandia National Laboratories) 


\section{Interfacial-Force Microscopy Probes The Mechanical Properties Of Single, Nanometer-Size Metal Grains KC 020102}

\section{Technical Achievement}

We have applied the Sandia Interfacial-Force Microscope (IFM) to the first study of the mechanical behavior of individual grains in polycrystalline Au films deposited on various substrates. The tungsten scanning-force probe has a nanometer scale tip and can be rastered across the surface to produce repulsive force images. In addition, the probe can be ramped into surface contact to measure the interfacial force necessary to produce local deformation. By a detailed analysis of scanning-force images and loading curves, we can obtain quantitative values for the modulus of elasticity and shear-stress threshold for permanent deformation of individual grains. These measurements are made possible by the unique capabilities of the self-balancing force sensor used in the IFM. No present scanning force microscopy or conventional nanoindentation instrument employs this unique sensor design. The sensor allows stable, quantitative interfacial force measurements without the mechanical instabilities present in all force sensors currently in use. Our single-grain analyses involved $200 \mathrm{~nm}$ polycrystalline Au films grown under differing conditions on several substrates. The results show that the measured elastic modulus for the grains varied considerably depending on the substrate ranging from $35 \mathrm{GPa}$ for glass to $110 \mathrm{GPa}$ for $\mathrm{Au}$ on $\mathrm{Si}(001)$ with a $10 \mathrm{~nm} \mathrm{Cr}$ adhesion layer. Single-crystal $\mathrm{Au}(111)$ yielded a value of $70 \mathrm{GPa}$, which is very close to the text-book value. However, the total deformation at threshold remained constant regardless of the substrate. These results indicate that the threshold strain for the creation of defects in the Au depends only on the local bonding interaction and not on the environment of the nanograins.

\section{Significance}

Considerable excitement has been generated in the material science community by the unique properties found for materials formed from nanometer scale building blocks. However, the ability to model and predict the properties of these materials has been hampered by the difficulty of making experimental measurements at the level of individual grains in these materials. Measurements that we have performed using the IFM will permit an accurate evaluation of the mechanical properties for nanophase materials as a function of the details of the fabrication processing. These results can be incorporated into modeling to permit fabrication to be fine tuned to tailor nanostrutcured materials for specific applications.

(Research performed by J. E. Houston and T. A. Michalske at Sandia National Laboratories.) 


\section{Direct Observation of Boron-Dopant Induced Effects on SiO2 CVD \\ KC 020303}

\section{$\underline{\text { Scientific Accomplishment }}$}

It is a well-known observation that the addition of dopant precursors can have pronounced effects on chemical vapor deposition processes. For example, the addition of boron and phosphorous precursors is known to increase the deposition rate of $\mathrm{SiO} 2$ using tetraethyl orthosilicate (TEOS). The study of this particular system provides a unique opportunity to advance the commercially important technology of boron-doped SiO2 CVD while expanding the rich science behind the effect of dopants on CVD growth. To this end, we have used FTIR and other surface sensitive spectroscopies to make the first direct observation of how the surface chemistry of such dopants affects CVD processes.

In our experiments we have used FTIR to measure the rate of TEOS chemisorption on $\mathrm{SiO}_{2}$ as a function of $\mathrm{SiOH}$ and $\mathrm{BOH}$ coverages over a wide range of temperatures and pressures. Our results show that at $300 \mathrm{~K}$ TEOS reacts with $\mathrm{BOH}$ at an "infinitely" greater rate than with $\mathrm{SiOH}$. In contrast, at $1000 \mathrm{~K}$ (using deuterated hydroxyls to distinguish hydroxyl consumption from hydroxyl reformation), TEOS reacts only twice as fast with $\mathrm{BOH}$ compared to $\mathrm{SiOH}$. In terms of activation energies, this result can be expressed as $E_{\mathrm{a}(\mathrm{TEOS}+\text { Boranol })}<<E_{\mathrm{a}(\mathrm{TEOS}+\text { Silanol) }}$.

\section{Significance}

These surface specific studies have demonstrated for the first time that the relative rate constants for TEOS + $\mathrm{BOH}$ and TEOS + SiOH have dramatically different temperature dependencies. They provide one of the first examples of the specific manner in which dopants on the surface can affect deposition kinetics. With these results we now understand why the deposition rates for the growth of boron-doped $\mathrm{SiO}_{2}$ from TMB and TEOS are sensitive to temperature as well as the relative concentrations of $\mathrm{BOH} / \mathrm{SiOH}$. This suggests that non-uniform $\mathrm{BOH}$ concentrations arising from effects such as gas-phase depletion may be a major cause of non-uniform growth. These insights are of considerable interest to the microelectronics industry from the standpoint of improving process reliability.

(Research performed by Michael E. Bartram and Harry K. Moffat, Sandia National Laboratories, New Mexico.) 


\title{
Band Structure Effects In Fullerene-Based Superconductors
}

\author{
KC 020202
}

\section{Scientific Achievement:}

We have demonstrated that the relationship between superconducting transition temperature and lattice constant, previously believed to be "universal" in the fullerene-based superconductors, in fact varies widely when non-alkali metal dopants are considered. The discovery of hightemperature superconductivity in alkali-doped fullerenes (crystals composed of $\mathrm{C}_{60}$ molecules) was a great surprise to the materials science community. Despite considerable effort, simply understandable "signposts" toward understanding the origin of superconductivity in these materials are few and far between. One such signpost, discovered through earlier pressure work here at Sandia and elsewhere, was the observation that, in fullerene crystals doped with alkali metals ( $\mathrm{Li}, \mathrm{Na}, \mathrm{K}$, etc.), the superconducting transition temperature seemed to be a "universal" function of the fullerene lattice constant. This observation suggested that only the fullerene lattice truly participated in the interaction leading to superconductivity, and the only function of the dopant atoms was to provide conduction electrons to participate in the phenomenon of superconductivity. Several theoretical descriptions based on this simple idea have been developed, and succeeded in obtaining "universal" curves qualitatively similar to those observed experimentally. If truly a general phenomenon, the existence of universal behavior in fullerenebased superconductivity would be a vital clue in understanding this new and baffling class of superconductors, as well as providing a useful tool for the design of superconductors for application.

Our collaborators at AT\&T Bells Labs have succeeded in synthesizing the alkaline-metal doped $\mathrm{Ca}_{5} \mathrm{C}_{60}$, whose superconductivity appears similar to that of the alkali-doped fullerenes. However, the change of transition temperature with applied pressure (and hence with reduced lattice constant) has the opposite sign and is an order of magnitude smaller than predicted by the "universal" curve obtained from the alkali-doped fullerene experiments. We have thus demonstrated that the "universal" behavior does not transcend oxidation number of the dopant species and that band structure effects cannot be ignored in understanding fullerene-based superconductivity.

\section{Significance:}

This achievement demonstrates that fullerene-based superconductivity is not simple. The models based solely on the properties of $\mathrm{C}_{60}$ molecules, the fullerene lattice, and the pool of electrons available from the dopant atoms are not adequate to describe this unique phenomenon. At a minimum, details of the electronic band structure must be considered in attempting an explanation. This result has wide-ranging implications for high-temperature superconductivity in general. The one common thread shared by numerous systems which exhibit some version of high-temperature superconductivity is the presence of weakly-linked structural units on a mesoscopic length scale. From this point of view, the fullerene-based superconductors are perhaps the simplest examples. Developing an understanding of superconductivity in these systems may thus lead to insights and even predictive models for the more complicated, but more useful, ceramic high-temperature superconductors.

(Research performed by J.E. Schirber at Sandia National Laboratories, in collaboration with D. Murphy and A.R. Kortan of AT\&T Bell Labs and J.E. Fischer of U. Penn.) 


\section{A New Understanding of Electron Cyclotron Resonance Plasmas Permits Controlled Hydrogen Treatment of Electronic Materials KC 020103}

\section{Technical Achievement}

We have employed new methods to achieve the first direct quantitative determination of the energies and fluxes of particles impinging from electron cyclotron resonance (ECR) hydrogen plasmas into solid targets. These studies employed three experimental techniques, two unique to the present program. First, a carbon-resistance particle detector developed at Sandia was used to measure the flux and energy of particles incident onto the solid target. Typical plasma conditions yielded $1.0 \pm 0.210^{16}$ particles $/ \mathrm{cm}^{2} / \mathrm{s}$ with an average energy of $35 \mathrm{eV}$. These results show that ECR plasmas are highly favorable for providing high fluxes at energies below the threshold for collision damage. Second, a Langmuir probe was used to determine the electron density and temperature as a function of magnetic field gradient. The optimum magnetic-field configuration was found to be a small field gradient, which strongly increased plasma density and diminished the unwanted transmission of microwave power through the plasma zone. Finally, the capability to predict hydrogen charging from measured plasma properties was demonstrated in experiments on $\mathrm{Si}$ using a new type of strong hydrogen sinks consisting of microscopic cavities. The accumulation of hydrogen at these sinks during plasma exposures was measured by ion-beam analysis; and using the incident-particle flux and energy given above, the rate of accumulation at the sinks was predicted to within a factor of 2 . In contrast, hydrogen charging rates in typical radio-frequency plasmas have been uncertain by as much as factors of 10 to 100 .

\section{Significance}

This work is the basis for well controlled and damage-free hydrogen introduction into materials having a small hydrogen solubility. An important application of this work is the hydrogen passivation of unwanted electrical states in semiconductors, and in particular the passivation of unwanted recombination centers in solar-cell materials. This ECR-plasma investigation has mitigated the typically large uncertainties in hydrogen-plasma treatment of materials, and has provided a methodology which is transferable to other types of plasma systems.

(Research performed by J. C. Barbour, W. R. Wampler and Craig A. Outten, Sandia National Laboratories) 


\section{Unified Model of Roughening Instability and Surface Evolution During Low Energy Ion Bombardment \\ KC 020103}

\section{Technical Achievement}

We have measured the temperature dependent roughening kinetics of Ge surfaces during low energy ion sputtering using in situ X-ray reflectivity. At elevated temperature, the surface develops an instability in which the roughness increases exponentially with time, much more rapidly than expected from a random sputter process. Atomic force microscope images indicate the surface develops a pronounced ripple topography during this roughening. At lower temperature, the surface is amorphized by the ion beam and rough surfaces become smoother during sputtering. The observed kinetics are explained quantitatively by a model which balances roughening by atom removal with smoothing by surface diffusion and viscous flow. The instability is related to the dependence of the sputter yield on the local surface curvature as proposed by Bradley and Harper (1988).

\section{$\underline{\text { Significance }}$}

Understanding and controlling surface roughness on the nanometer scale is becoming increasingly important in advanced processing of materials. Roughening kinetics in this regime have been particularly difficult to determine because of the lack of real-time in situ probes. The development of in situ X-ray reflectivity in this BES/MS program has allowed us to measure directly the real-time evolution of roughness during sputter processing. We have shown that the evolution of surface roughness can be understood with a linear model combining sputter roughening with surface transport. Our unified model quantitatively explains the kinetics of the roughening as well as the development of surface ripples. It is also the first quantitative test of the kinetics predicted by the Bradley-Harper instability mechanism. This work is a significant advance in our ability to understand and predict the evolution of surface morphology under low energy sputtering.

(Research performed by E. Chason, T.M. Mayer and A.J. Howard, Sandia National Laboratories.) 


\section{Direct Observation of Substitutional-Atom Trapping on a Metal Surface KC 020202}

\section{Scientific Accomplishment}

Direct observations of migrating surface atoms show conclusively that $\mathrm{Rh}$ adatoms on a $\mathrm{Rh}(100)$ surface are trapped in the four lattice sites adjacent to an Ir substitutional atom. The trap site is created by intentionally substituting an Ir atom on the surface for a Rh atom within the top layer of $\mathrm{Rh}$ atoms. In the temperature range from $250-320 \mathrm{~K}$, a $\mathrm{Rh}$ adatom migrates freely around the four sites neighboring the substitutional Ir atom, but does not move away. The energetics associated with the trap site have been determined from detailed investigations of the hopping rates to locations around and away from the impurity atom. A simple, pairwise-bond model with a stronger Ir-Rh bond compared to $\mathrm{Rh}-\mathrm{Rh}$ explains the observed trapping as well as differences in the electric fields required to remove $\mathrm{Rh}$ and $\mathrm{Ir}$ atoms from the surface. A second $\mathrm{Rh}$ adatom added to the trap site forms a stable dimer with the first and becomes even more strongly attached to the Ir impurity atom. This provides further evidence that the Ir substitutional atom serves as a nucleation site for homoepitaxial growth on the $\mathrm{Rh}(100)$ terrace.

\section{Significance}

The ability to achieve atomically smooth surfaces during crystal and epitaxial growth processes is becoming increasingly more important as scientists and engineers attempt to create new materials by controlling crystal growth at the atomic level. Although it is often assumed that layer-by-layer growth occurs when the rate of diffusion across single crystal terraces exceeds the rate of nucleation events, there is indirect evidence from macroscopic growth studies that isolated surface defects can serve as nucleation sites and inhibit uniform growth. Our results show unambiguously that substitutional impurity atoms do, in fact, trap migrating surface atoms. The quantitative energy barriers derived from this study are expected to provide valuable input into more realistic growth-simulation models that include the effect of impurity trapping. The results also suggest that by intentionally creating trap sites on a surface with impurity "seeds", it should be possible to alter the nucleation and growth of epitaxial layers, changing the growth process from "layer-by-layer" to "three-dimensional." Moreover, the direct observation and characterization of the energetics of impurity atom trapping will also serve to stimulate advanced theoretical modeling of adatom-impurity atom interactions, some of which has already begun.

(Research performed by G. L. Kellogg, Sandia National Laboratories) 


\section{Infrared Spectroscopy Of Adsorbates On GaAs Surfaces \\ KC 020303}

\section{$\underline{\text { Scientific Achievement }}$}

We have measured the vibrational properties of key adsorbates (e.g. $\mathrm{CH}_{2}, \mathrm{CH}_{3}, \mathrm{AsH}_{3}$ ) on $\mathrm{GaAs}$ surfaces using multiple internal reflection infrared spectroscopy (MIRIRS). We have been able to use the infrared spectrum as a fingerprint to determine the chemical identity of an adsorbate. By examining the polarization dependence of an adsorbate's vibrational modes, we can also determine many aspects of the adsorbate's bonding geometry with respect to the surface. For example, we have used MIRIRS to determine the local bonding geometry of an ordered adlayer of $\mathrm{CH}_{3}$ groups on GaAs. An ordered (1 X 2) structure is created by briefly exposing the GaAs $(100)$ surface to trimethylgallium (TMGa) at $350^{\circ} \mathrm{C}$. By examining the polarization dependence of the $\mathrm{CH}_{3}$ vibrational modes, we have found that the adsorbate is bonded exclusively to gallium atoms with the $\mathrm{Ga}-\mathrm{C}$ bond tilted $50 \pm 10^{\circ}$ from the surface normal.

\section{Significance}

Molecular precursors (e.g. TMGa, $\mathrm{AsH}_{3}$ ) are widely used as sources during the metal-organic chemical vapor deposition (MOCVD) of compound semiconductor thin films. Under many conditions the growing surface is covered with adsorbates that were once part of the molecular precursor (e.g. $-\mathrm{CH}_{3},-\mathrm{H}$ ). In order to fully understand how MOCVD works and to control the properties of the deposited films, we must determine the chemical and structural properties of these adsorbate-covered surfaces. The MIRIRS technique with polarization dependence is a powerful probe that allows us to address these issues. Our results for the (1X 2) $\mathrm{CH}_{3} / \mathrm{GaAs}(100)$ surface will serve as a benchmark for understanding the properties of other adsorbate-covered surfaces of compound semiconductors.

(Research performed by J. Randall Creighton, Sandia National Laboratories, New Mexico.) 


\section{B. Existing Programs (Continued)}

\section{Research Summaries}

1. Physics and Chemistry of Ceramics $\ldots \ldots \ldots \ldots \ldots \ldots \ldots \ldots \ldots \ldots \ldots . \ldots \ldots$

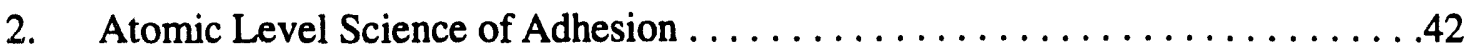

3. Energetic-Particle Synthesis and Science of Materials . . . . . . . . . . . 44

4. Advanced Growth Techniques and the Science of Epitaxy ..............46

5. Artificially-Structured Semiconductors $\ldots \ldots \ldots \ldots \ldots \ldots \ldots \ldots \ldots . \ldots 48$

6. Physics and Chemistry of Novel Superconductors $\ldots \ldots \ldots \ldots \ldots \ldots \ldots .50$

7. Tailored Surfaces and Interfaces for Materials Applications............52

8. Boron-Rich Solids . . . . . . . . . . . . . . . . . . . . . . . . . 54

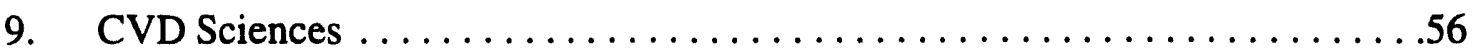

10. Synthesis and Processing of Nanoclusters for Energy Applications ..........59 


\section{Physics and Chemistry of Ceramics KC 020101}

Principal Investigators: R. Assink, C.J. Brinker, R.K. Brow, P.F. Green, A. J. Hurd, D.W. Schaefer, P.R. Schunk. R.W. Schwartz and J. Voigt

Goal: To identify and control the essential chemical and physical processes that determine the structure and properties of ceramic materials, especially those derived from solution.

Recent Highlights

- Technological Impact: Research from this project has led to a dozen CRADAs or other agreements with industry in the past 2 years (see Sec. V).

- Ligand Effects in Complex Ceramics: We determined the effects of precursor structure on the densification and crystallization behavior of zirconia thin films. By using appropriate ligands and ligand/metal ratios, we were able to vary the nature of the oligomeric species in solution from transparent to opaque. During film fabrication, the induced oligomer variations resulted in significant differences in aggregation behavior and consequently, large. controllable differences in the densification of films during heat-treatment.

- Film Formation: Based on a one-dimensional model for spin-coated sol gel films we now predict the evolution of pore size, network stress, and capillary stress development during film deposition. Second, we can predict the important effects of the gas phase on the drying film. Our novel two-phase approach uses parameters that are controlled in real experiments (e.g. room humidity, external air flow, etc.).

- Light scattering from ripplons was used successfully to determine the moment of gelation of a sol-gel film. The gel point is difficult to identify because the transition has no associated thermophysical or symmetry changes; it is strictly topologic. The dispersion of "ripplons" (thermally generated capillary waves) on the surface of a film is sensitive to near-surface viscosity, and by dynamic light scattering we were able to determine the gel point to within a few percent reduced time. As a byproduct, we got surface energy information.

- Porous Solids: Aerogels were prepared for the first time as thin films by a dip-coating process under ambient pressure and temperature conditions. Conventional dip-coating followed by low temperature pyrolysis led to the formation of films with refractive indices as low as 1.03 corresponding to about 95 volume percent porosity.

- Combined NMR and SAXS measurements elucidated the role of cyclic tetrasiloxanes in influencing the intermediate scale structure of silicate polymers. The incorporation of cyclic tetramers into the siloxane backbone results in extended structures characterized by low values of mass fractal dimension. The intermediate scale structure of the polymeric precursors in turn largely determines the microstructure of the resulting dried gels.

- We determined the mechanism of consolidation in aerogels. With increased annealing time, the solid phase grows in a powerlaw fashion while the size of the nanoscale voids remain unchanged. Concurrently, micron-scale domains develop (believel to be voids) that ultimately control defects in the fully sintered product.

- Dynamics of Glasses: We have shown, in mixed alkali oxide glasses, that the characteristic relaxation times that describe the response of the system to an external "stress" are comparable for nuclear spin relaxation and electrical conductivity relaxation. We further showed that the characteristic relaxation times for the response to mechanical stresses is two orders of magnitude slower than that due to electrical "stress". It appears that these observations are related to the degree of cooperativity that influences the dynamics of ion motion.

Future Directions

- Both pressure-sensitive dyes developed by the UCLA group are being tested in bulk pressure cells and the more promising one will be applied in sol-gel matrices to measure local capillary pressure. The adsorptive dye may be assessed in the future as a temperature probe to complement our chemical kinetics and evaporation models.

- Ripplon scattering will be used in conjunction with surface-acoustic-wave devices to seek the existence of nascent skin on drying films and fibers as predicted by some theories.

- The entire experimental and theoretical apparatus developed for films over the years is being converted to the study of ceramic fiber formation, which is critical to (among others) metal matrix composites.

- Since precursor structure and tendency toward condensation during deposition are key in defining thin film processing behavior, we will employ I70 NMR to look at the formation of metal-oxygen-metal bonds. For specimens already prepared from acetate and acetylacetone modified zirconium alkoxides, we will study organic burn-out using FT-IR spectroscopy and crystalline nature using X-ray diffraction to determine the relationships between pyrolysis, condensation reactions, film consolidation, and crystallization onset.

Program Quality

- 25 invited presentations, 4 invited review papers, 2 national meeting chairs, 7 symposium organizers/committees, 5 journal editors/associate editors, 2 NRC panelists, Chemtracts invited expert, Schwartzwalder Ceramics Prize. 2 MRS committees. 


\section{Physics and Chemistry of Ceramics KC 020101}

\section{Publications}

1. "The Short Range Structure of Ultraphosphae Glass", R. K. Brow. D. R. Tallant, J. J. Hudgens, S. Martin and A. D. Irwin. J. NonCryst. Solids, accepted, (1994).

2. "Relaxations in Mixed Alkali Metaphosphate Glass", P. F. Green, D. Sidebottom and R. K. Brow, J. Non-Cryst. Solids, accepted.

3. "Synthesis and Characterization of Group IV Metal Adamantanol Alkoxicdes as Potential PZT Precursors", T. J. Boyle, Aaron T. Pearson and R. W. Schwartz, Ceramic Transactions, Proc. Am. Ceram. Soc. PAC RIM Meeting, November, 1993, in press.

4. "In-Situ Fluorescence Imaging of Sol-Gel Thin Film Deposition", F. Nishida, J. M. McKiernan, B. Dunn, J. I. Zink, C. J. Brinker, and A. J. Hurd, J. Sol-Gel Sci. \& Tech., in press.

5. "Crystallization and Densification of Zirconia Thin Films Prepared by Sol-Gel Processing", R. W. Schwartz, J. A. Voigt, C. D. Buchheit and T. J. Boyle, Ceramic Transactions, Proc. Am. Ceram. Soc. PAC RIM Meeting, November, 1993, in press.

6. "Sol-Gel Strategies for Controlled Porosity Inorganic Materials", C. J. Brinker, R. Sehgal, S. L. Hietala, R. Deshpande, D. M. Smith, D. A. Loy, and C. S. Ashley, J. Membrane Sci., in press.

7. "Polymer Approach to Supported Silica Membranes", C. J. Brinker, R. Sehgal, N. Raman, P. R. Schunk, and T. J. Headley, Sol-Gel Sci. \& Tech., in press.

8. "Fundamentals of Sol-Gel Dip-Coating", C. J. Brinker and A. J. Hurd, submitted to J. de Phys. III.

9. "Pore Structure Evolution in Silica Gels During Aging and Drying 4, Varying Pore Fluid pH", P. J. Davis, R. Deshpande, D. M. Smith, C. J. Brinker, and R. A. Assink, J. Non-Cryst. Solids, in press.

10. "Relationships between the Structure and Properties of Silica Membranes and Films", C. J. Brinker, N. K. Raman, D. L. Logan, R. Sehgal, T. L. Ward, S. Wallace, and R. A. Assink, Polymer Preprints.

11. "Amorphous Sol-Gel Insulating Films", C. J. Brinker, W. L. Warren, M. N. Logan, and C. S. Ashley, Mat. Res. Soc. Symp. Proc. 284 (Pittsburgh, PA, 1993).

12. "Closed Porosity Aluminosilicate for Electronic Packaging Applications", S. L. Hietala, D. M. Smith, V. NM. Hietala, and C. J. Brinker, J. Mater. Res., 8 (1993) 1122.

13. "Power-Law Relaxation of Spin-1/2 Nuclei in Solids", R. A. Assink, M. B. Boslough, and R. T. Cygan, J. Magnetic Resonance, accepted.

14. "Solid State 207Pb CP-MAS NMR of Lead (II) Compounds for the Structural Study of Molecular Routes to Lead-Based Perovskite Phase Materials", A. D. Irwin, C. D. Chandler, R. A. Assink, and M. J. Hampden-Smith, submitted to Inorg. Chem. Comm. Ed.

15. "Densification and Crystallization of Zirconia Thin Films Prepared by Sol-Gel Processing", R. W. Schwartz, J. A. Voigt, C. D. Buchheit and T. J. Boyle, submitted to Ceramic Transactions.

16. "Synthesis of Novel Titanium and Zirconium Alkoxides as Precursors for Lead Zirconate Titanate (PZT) Thin Films", T. J. Boyle, A. T. Pearson, and R. W. Schwartz, submitted to Ceramic Transactions.

17. "Finite Element Modeling of Evaporation and Condersation During Sol-Gel Film and Fiber Formation", R. Schunk, A. J. Hurd, C. J. Brinker, and R. R. Rao 1993; Proceedings of the VIII International Conference on Finite Elements in Fluids, Barcelona. Pineridge Press.

18. "Free-Meniscus Coating Process, in Liquid Film Coating, Scientific Principles and their Technological Implications", R. Schunk, A. J. Hurd, and C. J. Brinker 1994; editors, P. M. Schweizer and S. F. Kistler, Chapman and Hall, New York.

19. "Finite Element Techniques for Calculation of Condensation and Evaporation Processes", R. Schunk and R. R. Rao, Int. J. Numer. Meth. Fluids. (in press).

20. "X-Ray Scattering Of Smectic Ordering In A Silica Aerogel", T. Bellini, N. A. Clark, R. M. Malzbender B N. Thomas, A. G. Rappaport. C. D. Muzny and D. W. Schaefer, Phys. Rev. Lett. 71, 3505 (1993) 
21. "Structure And Topology Of Silica Aerogels During Densification", D. W. Schaefer, B. J. Olivier, and C. S. Ashley, G. Beaucage, D. Richter, B. Farago, B. Frick and D. A. Fishcher, Journal of Noncrystalline Solids, in press (1994)

22. "General Routes To Porous Metal Oxides Via Inorganic And Organic Templates", C. Roger, D. W. Schaefer, G. B. Beaucage and M. J. Hampden-Smith, J. of Sol-Gel Sci. \& Tech., in press (1994).

23. "Structure of Mesoporous Aerogels", D. W. Schaefer, MRS Builetin, 19 (4), xxx, (1994).

24. "Engineered Porous Materials", D. W. Schaefer, MRS Bulletin, 19 (4), xxx, (1994)

25. "Structural Studies of Complex Systems using Small-Angle Scattering: A Unified Guinier/Power-Law Approach", G. Beaucage, and D. W. Schaefer, J. Non-Cryst. Solids., in press (1994).

26. "Phase Behavior of the Liquid Crystal 8CB in a Silica Aerogel", T. Bellini, N. A. Clark, C. D. Muzny, L. Wu, C. W. Garland, D. W. Schaefer and B. J. OPliver, submitted to J. Appl. Phys.

27. "Characterization of Porosity in Ceramic Materials by Small-Angle Scattering: VycorTM Glass and Silica Aerogel", D. W. Schaefer, R. K. Brow, B. J. Olivier, T. Rieker, G. Beaucage, L. Hrubesh and J. S. Lin, in Modern Aspects of Small-Angle Scattering, edited by Harry Brumberger, (Kluwer Academic Publishers, Dordrecht, 1994). 


\title{
Atomic Level Science of Adhesion KC 020102
}

\author{
Principal Investigators: T. A. Michalske, J. E. Houston, N. D. Shinn J. S. Nelson and P. J. Feibelman
}

Goal: To understand in atomic detail the nature of the physical and chemical interactions that control the binding of solid surfaces and to use this understanding to: 1) develop predictive models for adhesive bond stability and 2) tailor material surfaces and interfaces for optimized adhesion and lubrication properties.

\section{Recent Highlights}

- Nanomechanical Properties: The extraordinary force and displacement control of the Interfacial Force Microscope has been used to make the first quantitative measurements of the mechanical properties of nanometer-scale grains in polycrystalline films.

Plastic Deformation Threshold: We have quantitatively measured the shear-stress threshold for plastic deformation of nanometer size grains in polycrystalline Au films. We showed that while the shear stress at threshold is sensitive to thin film substrate interactions, the shear displacement at threshold is constant. These results indicate that the crystal lattice stability in nanometer-scale structures depends only on the local bonding interactions. This type of information is critical to understanding the enhanced mechanical properties of nanophase materials.

Grain Boundary Sliding: By mechanically probing individual grains in polycrystalline Au films we have been able to measure the shear stress required for grain boundary sliding of $50 \mathrm{MPa}$ at room temperature. No previous measurements have directly observed the stress-strain behavior of intergranular motion. These measurements will provide new data for models describing metal forming and superplastic deformation.

- Monolayer Lubricants: Self-assembling alkane thiol monolayers act as excellent lubricants on Au surfaces by preventing direct metal/metal contact. We have found that the detailed load response for these lubricating layers shows three distinct regions: initially the films appear soft and the molecules compact by leaning, higher loads show an increase in film stiffness which results from, according to recent theoretical results, head-group structural changes, and finally the films become fully compressed and very hard. These results demonstrate how detailed nanomechanical measurements can be used to evaluate molecular-scale lubrication models.

- Adhesive Bond Formation: We have made the first measurements of the electrostatic component of the adhesive bond. These measurements were made between Au probe and Au substrate surfaces both coated with alkane thiol self-assembling monolayers. The electrostatic component of the adhesive bond results from the dipole-dipole attraction between these films when "end groups" having different polarity are incorporated in the two films. Not only are such measurements important because they yield information concerning the chemical nature of the surfaces, but because they permit an evaluation of interfacial bonding which is presently thought to dominate oxide/oxide and oxide/metal adhesion.

- Technology Transfer: We are in our second year of three DP-funded CRADA agreements for a technology transfer initiative involving Sandia, Digital Instruments, AT\&T Bell Laboratories, and the University of New Mexico. The goal of this program which is based on our development of the Interfacial Force Microscope (IFM) under the BES program is to develop a commercial version that can be used to provide three dimensional images and measurements of microfabricated structures such as microelectronics circuits or precision machined materials. The increased operating stability of the IFM design over the conventional Atomic Force Microscope will greatly broaden the range of application of this scanning probe microscopy in materials and biological studies.

\section{Future Directions}

This program will continue to focus on namometer-scale measurements of interfacial adhesion, the development of theoretical models to address the chemical stability of the adhesive interface, and macroscopic measurements to test microscopic predictions. We will tailor the probe tip and substrate surfaces with self-assembled monolayer films so that we can examine in a controlled fashion the role of surface chemical groups on the formation and stability of the adhesive bond. To facilitate these measurements, the IFM will be brought into an ultra-high vacuum apparatus so that adhesion events can be examined on clean and adsorbate covered surfaces and the results compared with first principles atomistic simulations. We will also use the IFM to probe the mechanical response of small-scale structures so that we can better understand the behavior of materials near stress singularities and nanoscale composite materials.

\section{Program Quality}

- 7 publications, 10 invited presentations (MRS, 2-ACS, 2-Gordon Conf., APS, ASME)

- Invited manuscript in J. Adhesion Sci. and Technol.

- 1993 BES/Materials Science Award for Significant Implications to DOE Technologies in Metallurgy and Ceramics. 


\section{Atomic Level Science of Adhesion \\ KC 020102}

\section{Publications}

1. "The Mechanical Response of Gold Substrates Passivated by Self-Assembling Monolayer Films", R. C. Thomas, J. E. Houston, T. A. Michalske and R. M. Crooks, Science 259, 1883 (1993).

2. "The Nanometer-Scale Mechanics of Gold Films", P. Tangyunyong, R. C. Thomas, J. E. Houston, T. A. Michalske, R. M. Crooks and A. J. Howard, Phys. Rev. Lett. 71, 3319 (1993).

3. "Substrate Effects on the Nanometer-Scale Mechanics of Gold Films", P. Tangyunyong, R. C. Thomas, J. E. Houston, T. A. Michalske, R. M. Crooks and A. J. Howard, J. Adhesion Sci. and Technol. (In Press).

4. "Chemically-Sensitive Interfacial Force Microscopy: Contact Potential Measurements of Self-Assembling Monolayer Films", R. C. Thomas, P. Tangyunyong, J. E. Houston, T. A. Michalske and R. M. Crooks, J. Phys. Chem. (In Press).

5. "The Role of Structural Relaxations in the Fracture of Vitreous Silica", T. A. Michalske, W. L. Smith, J. E. Houston, J. Appl. Phys.

6. "Soft X-ray Photoelectron Spectroscopy of Bi-metallic Interfacial Bonding", N. D. Shinn, Materials Research Society Proceedings, 307, 167 (MRS, 1993)

7. "Oxidation of W(110): Valence-Band and W(4f) Core-level Spectroscopy", N. D. Shinn, Surface Science (In Press). 


\section{Energetic-Particle Synthesis and Science of Materials KC 020103}

Principal Investigators: J. C. Barbour, R. J. Bourcier, J. S. Custer, M. T. Dugger, D. M. Follstaedt, J. A. Knapp, S. M. Myers, C. H. Seager, H. J. Stein, and W. R. Wampler

Goals: Use energei:c beams and plasmas to create superior new materials and to achieve fundamental understanding of the atomic procesies, defects, and microstructures that govern materials processing and properties.

\section{Recent Highlights}

- Metal-atom trapping at cavities in Si: The internal surfaces of cavities in Si formed by He ion implantation and annealing were shown to bind $\mathrm{Cu}$ and $\mathrm{Ni}$ impurities substantially more strongly than metal-silicide precipitation. Cavities provide a new approach to impurity gettering that can reduce residual impurity concentrations to much lower levels and dissolve silicide particles from critical locations within devices.

- Tailored properties of ECR-plasma-grown Si-oxynitrides: Studies of Si-N-O-H films grown on GaAs and InSb from electron-cyclotron-resonance (ECR) plasmas have yielded new mechanistic understanding of the influence of plasma and substrate conditions on key properties including stress, adhesion, surface roughness, interfacial mixing, and the bonding and electrical-passivation effects of $\mathbf{H}$. This knowledge provides a basis for more effective and controlled surface passivation of compound semiconductors, currently an important technological limitation.

- Gigapascal-strength Al-O films grown by pulsed laser deposition: Aluminum-O alloys containing 33 volume \% of nanometer-size $\mathrm{Al}_{2} \mathrm{O}_{3}$ precipitates were synthesized by pulsed laser deposition (PLD). We had previously formed this highly refined microstructure by ion implantation and had demonstrated that it has gigapascal flow stresses similar to those of high-strength steels as well as greatly enhanced tribological properties. Synthesis by PLD, a process that is scaleable to larger dimensions and not subject to the inherent thickness limitations of ion implantation, makes accessible a much wider range of technological applications.

- Electronic states at the Si surface: Cavities formed within Si by He ion implantation and annealing were used to characterize band-gap electronic levels associated with surface dangling bonds and to determine the intlisence of these levels on carriers in the adjacent Si matrix. By examining internal rather than external surfaces, it was possible for the first time to bring to bear such powerful electrical probes as deep-level transient spectroscopy, capacitance versus voltage, and conductance versus temperature.

- Predictive understanding of plasma injection of $\mathrm{H}$ into $\mathrm{Si}$ : The near-surface concentration of $\mathrm{H}$ in $\mathrm{Si}$ during exposure to ECR $\mathrm{H}$ plasmas was experimentally determined and quantitatively interpreted in terms of plasma parameters. Plasma charging is widely used to inject $\mathrm{H}$ into semiconductors for passivation, but the controlling near-surface boundary condition had not been measured or quantitatively predicted until the present study.

- $\underline{H}$ location on Si(100): Transmission-ion-channeling analysis determined the lattice position of $\mathrm{H}$ in the monohydride state on the (100) Si surface to a substantially higher accuracy than had previously been possible. This represents a significant advance in characterization of the technologically important $2 \times 1 \mathrm{H}$ termination.

\section{Future Directions}

- Beam synthesis of superior new materials using energetic beams and plasmas

- Nanocavities in Si and compound semiconductors for impurity gettering, dislocation trapping, and electrical isolation.

- Low-temperature oxynitrides grown from ECR plasmas for surface passivation and enhanced mechanical properties.

- Gigapascal-strength Al-O and Al-N alloys synthesized using ion implantation, PLD, and ECR plasmas.

- Ion-beam studies of fundamental atomic processes, defects. and microstructures

- Trapping mechanisms for detrimental metallic impurities in semiconductors.

- Injection and bonding of $\mathrm{H}$ in semiconductors as related to passivation of defects, surfaces, and impurities.

- Precipitation hardening and plastic flow in $\mathrm{Al}-\mathrm{Al}_{2} \mathrm{O}_{3}$ and $\mathrm{Al}$-AIN nanoscale alloys.

- Electronic and optical properties of bare and adsorbate-terminated internal surfaces in semiconductors.

\section{Program Quality}

- Requests from 12 semiconductor companies and SEMATECH for information on inetal-atom trapping by cavities in $\mathrm{Si}$.

- Exploratory application of O-implanted Al by a commercial firm.

- Completed CRADA with Charles Evans and Associates on ion-beam analysis of light elements.

- 3 invited papers and presentations, 13 publications. 


\section{Energetic-Particle Synthesis And Science Of Materials KC 020103}

\section{Publications}

\section{Work Performed under Present BES Program}

1. "Ion-Beam Synthesis Of Novel Materials", D. M. Follstaedt and S. M. Myers, Materials Technology (in press).

2. "Binding Of Copper To Nanocavities In Silicon", S. M. Myers, D. M. Follstaedt, and D. M. Bishop, Proc. 17th Inter. Conf. on Defects in Semiconductors, Gmunden, Austria, July 18-23, 1993 (in press).

3. "Binding Of Copper And Nickel To Cavities In Silicon Formed By Helium Ion Implantation", S. M. Myers, D. M. Follstaedt, and D. M. Bishop. Proc. 1993 Fall MRS Meeting (in press).

4. "Microstructures Of Si Surface Layers Implanted With Cu", D. M. Follstaedt and S. M. Myers, Proc. 1993 Fall MRS Meeting (in press).

5. "The Electrical Properties Of Helium-Implantation-Produced Nanocavities In Silicon", C. H. Seager, S. M. Myers, R. A. Anderson, W. L. Warren, and D. M. Follstaedt, Phys. Rev. B. (submitted).

6. "The Structural, Chemical, And Electrical Properties Of He-Implantation-Induced Nanocavities In Si", C. H. Seager, S. M. Myers, R. A. Anderson, D. M. Follstaedt, and H. J. Stein, in Proc. 3rd Workshop on the Role of Point Defects / Defect Complexes in Silicon Device Fabrication, Vail, CO, Aug. 16-18, 1993, edited by B. Sopori (in press).

7. "Tribological Improvement Of Pure Aluminum By Oxygen Implantation", M. T. Dugger, R. J. Bourcier, D. M. Follstaedt, and S. M. Myers, Tribology International (submitted).

8. "ECR Plasma Synthesis Of Silicon Nitride Films On GaAs And InSb", J. C. Barbour, M. L. Lovejoy, C. I. H. Ashby, A. J. Howard, J. S. Custer, and R. J. Shul, Proc. 1993 Fall MRS Meeting (in press).

9. "Hydrogen Uptake Into Silicon From An Ecr Plasma", W. R. Wampler and J. C. Barbour, J. Vac. Sci. Technol. (submitted).

10. "Vibrational Mode For Nitrogen In Zinc Selenide". H. J. Stein, Appl. Phys. Lett. (submitted).

11. "Hydrogen Introduction And Hydrogen-enhanced Thermal-donor Formation In Silicon", H. J. Stein and S.-K. Hahn, J. Appl. Phys. (in press).

Work Performed Jointly with Other Programs

12. "Ion-assisted Pulsed Laser Deposition Of Cubic Boron Nitride", T. A. Friedmann, P. B. Mirkarimi, D. L. Medlin, K. F. McCarty, E. J. Klaus, D. R. Boehme, H. A. Johnsen, M. J. Mills, and D. K. Ottesen, and J. C. Barbour, J. Appl. Phys. (submitted).

13. "Nucleation And Growth Of Cubic Boron-Nitride Films Produced By Ion-Assisted Pulsed Laser Deposition", T. A. Friedmann, D. L. Medlin, P. B. Mirkarimi, K. F. McCarty, E. J. Klaus, D. R. Boehme, H. A. Johnsen, M. J. Mills, D. K. Ottesen, and J. C. Barbour, Proc. 1993 Fall MRS Meeting (in press). 


\title{
Advanced Growth Techniques And The Science Of Epitaxy KC 020103
}

\author{
Principal Investigators: E. Chason, J.Y. Tsao, J.A. Floro, T. Drummond, B.S. Swartzentruber, H.J. Stein and S.T. \\ Picraux
}

Goal: To advance our understanding of epitaxial crystal growth and the fundamental processes governing it. Advances will include the development of novel growth techniques, in situ diagnostics and models leading to an integrated understanding of the interactions among various surface processes which control growth.

\section{Recent Highlights}

- CRADA with Motorola for SiGe/Si Heteroepitaxy - We have recently established a CRADA with Motorola for optimization of SiGeand SiGeC heterostructures for advanced electronic devices. Many of the advanced diagnostics and growth techniques were developed in our BES programs. Funding for the CRADA is obtained through DOE/DP.

- Roughening Instability and Unified Model of Ion-Induced Surface Morphology Evolution - Using in situ X-ray reflectivity, we have observed a roughening instability during low energy ion sputtering of Ge at elevated temperture and ion-induced surface smoothing at lower temperature. We have developed a unified model that quantitatively explains these kinetics as a balance between roughening by sputter removal of material and smoothing by surface diffusion and viscous flow.

- Real-Time Observation of Surface Defect Kinetics on Si(001) - Using our recently developed variable-temperature scanning tunneling microscope, we have observed the real-time formation and highly anisotropic migration of vacancies on the $\mathrm{Si}(001)$ surface at elevated temperatures; the fast diffusion direction is parallel to surface dimer rows. We have also measured the rate of atomic rearrangements at steps and determined an activation barrier of $1.4 \mathrm{eV}$.

- Stability of Si-C and Si-Ge-C Alloy Layers Grown by Solid Phase Epitaxy - Detailed thermal stability studies of $\mathrm{Si}-\mathrm{C}$ alloys grown by solid phase epitaxy have shown that $\mathrm{C}$ is lost from substitutional sites with the activation energy for $\mathrm{C}$ difusion in $\mathrm{Si}$, and that the $\beta$-SiC precipitates form without an activation barrier. Similar $\mathrm{C}$ loss processes occur in Si-Ge-C alloys, independent of strain compensation.

- Strain Relaxation in Linearly-and Step-Graded Buffers - Relaxation of both linearly- and step-graded InAlGaAs buffers on GaAs substrates saturates at values below those predicted by equilibrium theory without dislocation interactions. These results indicate that dislocation-dislocation interactions are important in understanding relaxation and that epilayers must be matched to the incompletely relaxed buffer to avoid formation of additional dislocations in critical device layers.

- Effect of Surface Treatments on Si Wafer Roughness - Control of nano-scale surface roughness is becoming increasingly important in advanced materials processing. We have used in situ X-ray reflectivity to characterize surface roughness of $\mathrm{Si}$ wafers after various cleaning treatments with resolution of better than $0.3 \AA$.

\section{Future Directions}

Scientific and technological issues in heteroepitaxy will remain a major focus of the advanced growth program. New in situ diagnostics such as RHEED characterization of strain will be developed to study relaxation kinetics in real time and novel techniques for minimizing strain-induced defects such as graded-buffers will continue to be explored. The recent completion of our MBE system for growth of ternary alloys will allow us to extend these techniques to group IV alloys. Si-Ge-C alloys will be grown at various compositions to determine the effect of strain and carbon content on structural and electronic properties. Along with e-beam deposition, the ECR plasma source will be used to deposit energetic $\mathrm{C}$ to attempt to minimize $\mathrm{SiC}$ formation. We will apply our understanding of the roughening instability during sputtering to similar instabilites that develop during strained heterolayer growth. Studies of surface defect kinetics will continue using high temperature STM and RHEED. New STM methods based on patternrecognition will be developed to enable the identification of different chemical species on the surface. A new highly collimated electron gun will be tested in collaboration with FEI for surface imaging in a scanning-RHEED mode. The CRADA with Motorola will complete a feedback loop that will enhance the incorporation of these advanced growth techniques and diagnostics into real processing environments.

Program Quality

14 publications, 16 presentations ( 3 invited) 


\section{Advanced Growth Techniques and The Science of Epitaxy KC 020103 \\ Publications}

1. "Roughening Instability And lon-Induced Viscous Relaxation Of $\mathrm{SiO}_{2}$ Surfaces", T.M. Mayer, E. Chason and A.J. Howard, J. Appl. Phys., submitted.

2. "In Situ X-Ray Reflectivity Measurements Of Thin Film Structural Evolution", E. Chason and M.K. Chason, J. Vac. Sci. Technol., in press.

3. "Roughening Instability And Evolution Of Ge(001) Surface During Ion Sputtering", E. Chason, T.M. Mayer, B.K. Kellerman, D.T. McIlroy and A.J. Howard, Phys. Rev. Lett., submitted.

4. "Intra-Cascade Surface Recombination Of Point Defects During Ion Bombardment Of Ge (001)", J.A. Floro, B.K. Kellerman, E. Chason, S.T. Picraux, D.K. Brice and K.M. Horn, Mat. Res. Soc. Symp. Proc., submitted.

5. "Surface Defect Production On Ge (001) During Low Energy Ion Bombardment", J.A. Floro, B.K. Kellerman, E. Chason, S.T. Picraux, D.K. Brice and K.M. Horn, J. Appl. Phys, submitted.

6. "Roughening Instability And Viscous Relaxation During Ion Sputtering", E. Chason, T.M. Mayer and A.J. Howard, Mat. Res. Soc. Symp. Proc., submitted..

7. "Oxygen Roughening Of Ge(001) Surfaces", K.M. Horn, E. Chason, J.Y. Tsao, J.A. Floro and S.T. Picraux, J. Appl. Phys., submitted.

8. "Stability And Precipitation Kinetics In $\mathrm{Si}_{1-y} \mathrm{C}_{\mathbf{y}} / \mathrm{Si}$ and $\mathrm{Si}_{1-x-y} \mathrm{Ge}_{\mathrm{x}} \mathrm{C}_{y} / \mathrm{Si}$ Heterostructures Prepared By Solid Phase Epitaxy", J.W. Strane, S.T. Picraux, H.J. Stein, S.R. Lee, J. Candelaria, D. Theodore and J.W. Mayer, Mat. Res. Soc. Symp. Proc., submitted

Work performed jointly with other programs

9. "Symmetry And Stability Of Solitary Dimer Rows On Si(100)", P. Bedrossion and E. Kaxiras, Phys. Rev. Lett. 70, 2589 (1993).

10. "Strain Relaxation And Surface Roughness As A Function Of Growth Temperature In Linearly-Graded $\operatorname{In}_{\mathbf{x}} A \mathrm{Al}_{1}$. ${ }_{x}$ As Buffers", J.A. Olsen, E.L. Hu, S.R. Lee, I.J. Fritz, A.J. Howard, B.E. Hammons, and J.Y. Tsao, Mat. Res. Soc. Symp. Proc., 1993, submitted.

11. "Strain Relaxation, Tilting, And Surface Roughness In Compositionally Graded AlInAs Buffers", J.A. Olsen, E.L. Hu, S.R.Lee, I.J. Fritz, A.J. Howard, B.E. Hammons, and J.Y. Tsao, J. Appl, Phys.

12. "MBE Grown III-V Strain Relaxed Buffer Layers And Superlattices Characterized By Atomic Force Microscopy", A.J.Howard, I.J. Fritz, T.J. Drummomd, J.A. Olsen, B.E. Hammons, S.R.Kurtz, and T.M. Brennan, J. Vac Sci. Technol., submitted.

13. "Variable-Temperature Stm Measurements Of Step Kinetics On Si(001)", N. Kitamura, B.S. Swartzentruber, M.G. Lagally and M.B. Webb, Phys. Rev. B 48, 5704 (1993).

14. "Strained-Layer Superlattice Technology For Vertical-Cavity Optoelectronic Modulators At Near-Infrared Wavelengths", I.J. Fritz, J.A. Olsen, A.J. Howard, T.M. Brennan, B.E.Hammons and G.A. Vawter, IEEE Journal of Quantum Electronics, submitted. 


\title{
Artificially-Structured Semiconductors KC 020105
}

\author{
Principal Investigators: P. L.Gourley, E. D. Jones, S. K. Lyo, J. S. Nelson, R. P. Schneider, Jr., and M.B. Sinclair
}

Goals: Investigate fundamental structural, optical, and electronic properties of strained-layers, quantum wells, heterostructures, artificially layered and patterned semiconductors grown by molecular beam epitaxy and metal organic vapor phase epitaxy. Identify new artificial materials with potential for high scientific and technological impact

\section{Recent Highlights:}

- Photonic Lattices Give 2-Dimensional Control of Photon Proparation - 2-dimensional photonic lattices were fabricated with e-beam lithography as honeycomb nanostructures by etching $250 \mathrm{~nm}$ air holes in an AlGaAs epitaxial layer. Using laser spectroscopy, we have shown that photons injected into the layer are inhibited from propagating along selected lattice directions. These photons which satisfy a Bragg condition are scattered normally out of the plane, demonstrating 2-dimensional control of photon propagation. The results also demonstrate that these new structures are useful for photon confinement in high efficiency light sources.

- Theory and Experiment Provide Estimates of Direct to Indirect Crossover in InAlGaP - We have used first principles self-consistent pseudopotential band structure techniques, in combination with pressure dependent photoluminescence measurements to determine the $A$ l concentration dependence of the $\Gamma-X$ crossing in $\operatorname{In}_{y}\left(\left.A\right|_{x} G a_{1-x}\right)_{1-y} P$. A special quasi-random structure is used to model local disorder in the quaternary alloy. The calculations predict a direct to indirect crossing at an $A 1$ concentration of $x=58 \% \pm 5 \%$. This value is slightly larger than the experimental estimates of $50 \%$. that we have determined at room temperature. These estimates provide upper limits on the direct bandgap in these alloys which are being considered for visible light emitters.

- Quantum Size Effects are Observed in New UnicompositionaL Quantum Wells - We have used metalorganic vapor phase epitaxy (MOVPE) to grow unicompositional disorder-order quantum well (QW) structures. The $\mathrm{QW}$ layer is composed of highly ordered GalnP and the barriers are highly disordered GalnP as revealed in transmission electron dark-field micrographs. Low-temperature photoluminescence from the structures exhibit relatively broad emission peaks, and emission energy increasing as QW thickness decreases. These results demonstrate the usefulness of order-disorder phenomena for band structure engineering.

- Femtosecond Spectroscopy Reveals Carrier Relaxation Rates in Fractal Ouantum Wells - We have performed ultra fast photoinduced absorption measurements of carrier relaxation in AIGaAs fractal quantum wells. In these layered structures, hundreds of localized electronic states are created by varying the Al composition between layers in a fractal sequence. After absorption of $100 \mathrm{fs}$ laser pulses, bleaching signals were monitored to track the electron-hole population in these localized states. We find that thick layers $(-200-400 \AA)$ are effective in localizing carriers in high energy states. This localization promotes broadband light emission in these novel materials.

Future Direction:

Development of heterostructures, fractal quantum wells, and short period and unicompositional superlattices in the InAIGaP materials system. Fabrication and photon propagation studies in 2-dimensional photonic lattices with complete photonic bandgaps for high efficiency light sources. Continued study of order/disorder effects on optical and electronic properties with collaborations with NREL on aspects relevant to photovotaics.

\section{Program Quality:}

- 1993 Amer. Physical Society International Prize for New Materials.

- 1993 BES Materials Division Award for Sustained Outstanding Research.

- 10 invited talks, 3 invited review articles and 12 publications. 


\section{Artlficially-Structured Semiconductors \\ KC 020105}

\section{Publications}

1. "Optical Properties of 2-dimensional Photonic Lattices Fabricated as Honeycomb Nanostructures in Compound Semiconductors", P. L Gourley, J. R. Wendt, G.A. Vawter, T.M. Brennan, and B. E. Hammons, Appl. Phys. Lett., (in press).

2. “Optical Properties of Honeycomb Photonic Lattices", P. L. Gourley, J. R. Wendt, G. A. Vawter, T. M. Brennan, and B. E. Hammons, Proceedings of the Quantum Electronics and Laser Science Conference, May 2-7, 1993, Baltimore, MD.

3. "Microstructured Semiconductors", P. L. Gourley, Comments on Condensed Matter Physics, invited review, submitted August, 1993.

4. "Semiconductor Microcavities", P.L. Gourley, J. R. Wendt, G. A. Vawter, M. E. Warren, T. M. Brennan, and B. E. Hammons, Proceedings of the International Society for Optical Engineering Conference OE/LASE94, submitted December, 1993.

5. "Exciton Magnetoluminescence Studies in Ordered and Disordered InGaP Semiconductor Alloys", E. Jones, D. M. Follstaedt, S. K. Lyo, and R. P. Schneider, Matl. Res. Soc. Symp. Proc. 281, 61, 1993

6. "Theory of Magnetic-Field Dependent Alloy Broadening of Exciton Photoluminescence Linewidths in Semiconductor Alloys", S. K. Lyo, Phys. Rev. B 48, 2152 (1993)

7. "Effect of Exciton Localization and Delocalization on Magnetic-Field-Dependent Photoluminescence Linewidths in Semiconductors", S.K. Lyo, E. D. Jones and S. R. Kurtz, J. Lumin. in press

8. "Ordering and Phase Separation in MOCVD InGap Alloys and Unicompositional Quantum Wells", E. D. Jones, D. M. Follstaedt, R. P. Schneider, Jr., Proceedings Symposium M.. Materials Research Society, November 29. December 3, 1993, Boston, MA.

9. "Excitonic Transitions in InGaP/AIGalnP Strained Quantum Wells", R. P. Schneider, E. D. Jones, J. A. Lott, Appl. Phys. letter 631240 (1993)

Work performed Jointly with Other Programs

10. "Plane Wave Electronic Structure Calculations on a Parailel Supercomputer", J. S. Nelson, S. J. Plimpton and M. P. Sears, Physical Review B47, 1795 (1993)

11. "Submicrometer Control of 2-Dimensional to 2-Dimensional Magnetotunneling in Double Well Heterostructures", J. A. Simmons, S. K. Lyo, J. F. Klem, M. E. Sherwin and J. R. Wendt, Phys. Rev. B 47, 15741 (1993)

12. "Linear Response Theory of Inter-Qantum-Well Tunneling in a Double-Well Structure with In-Plane Magnetic Fields", S. K. Lyo and J. A. Simmons, J. Phys. Condens. Matter 5, L299, (1993) 


\title{
Physics and Chemistry of Novel Superconductors KC $020202 \&$ KC 020205
}

\author{
Principal Investigators: B. Morosin, J.E. Schirber, M.P. Siegal, E.B. Stechel, and E.L. Venturini
}

Goals: To develop a fundamental understanding of the physics and chemistry of novel superconductors, emphasizing study of intrinsic properties, structure-based control of the transport and magnetic properties, and investigation of prototype device configurations.

\section{Recent Highlights:}

- Technological Interactions: Work in this program has led to a DP-funded CRADA with Conductus on continued development of the confocal resonator, a microwave-based tehnique which allows high resolution scanning of the microwave properties of a surface. Another CRADA, for developing synthesis and processing techniques for the Tl-based thin film superconductors, is currently under negotiation with DuPont. In addition, this program interacts closely with the Superconductor Technology Program funded at Sandia by DOE-EERE.

- Improved TIBaCaCuO thin film precursors: Tl-free $\mathrm{Ba}-\mathrm{Ca}-\mathrm{Cu}-\mathrm{O}$ precursor films are co-sputtered, resulting in a homogeneous mixture of oxides. This differs both chemically and physically from the heterogeneous metal layers previously used. When $\mathrm{Tl}$ is added using the traditional closed crucible technique, single-phase Tl-2212 and Tl2223 films have been obtained. These films are smoother and more uniform, and have much higher critical current densities in high magnetic fields.

- Development of Equilibrium Processing for TlBaCaCuO thin films: Two-zone processing of T1-HTS materials has been extended to include thin films. This is the only controlled and scalable technique for growth of single-phase and highly-epitaxial films, and is expected to completely replace the closed crucible technique. Initial trials have produced single-phase films of the 2212 and 1212 high- $\mathrm{T}_{\mathrm{c}}$ T7-based phases. Such films will be used to support both novel device programs that require weak pinning such as the Sandia-BES superconducting flux-flow transistor, as well as for fundamental superconductivity studies.

- Single Crystal Studies: We have extended our knowledge of the growth of Tl-containing single crystals, obtaining optimum $\mathrm{T}_{\mathrm{c}}$ 's with narrow transitions, and continue to examine structure/stoichiometry/doping relationships to test our $\mathrm{Tl}^{1+}-\mathrm{Tl}^{3+}$ equilibrium model. Studies on systems doped with $\mathrm{Pb}$ or $\mathrm{Sr}$ were initiated, and superconducting crystals of $\mathrm{TlSr}_{2} \mathrm{CaCu}_{2} \mathrm{O}_{x}$ were successfully grown and studied.

Our unique high pressure/high temperature oxygen processing capabilities continue to provide high quality single crystals of $\mathrm{La}_{2} \mathrm{CuO}_{4+d}$ superconductors for studies of phase separation and microscopic structural studies. NMR, $\mathrm{NQR}$, transport, and $\mathrm{dT} / \mathrm{dP}$ measurements as a function of temperature reveal details of the oxygen distribution and mobility, and doped-hole segregation in these model materials.

- Vortex Pinning Energy Magnetic relaxation for highly crystalline $\mathrm{YBa}_{2} \mathrm{Cu}_{3} \mathrm{O}_{7}$ films shows that the vortex pinning energy is significantly increased following irradiation by high-energy Xe ions. As a result, the dissipation from vortex motion at a current density of $10^{6} \mathrm{~A} / \mathrm{cm}^{2}$ is reduced by a factor of 50 at $77 \mathrm{~K}$. The cluster defects produced by high-energy $\mathrm{Xe}$ irradiation are more effective in pinning vortices than the point defects produced by $\mathrm{H}$ ions.

- Fullerene-based superconductors Our pressure studies of superconducting alkali-doped fullerenes helped establish a "universal" $T_{c}$ vs lattice constant curve as predicted by several theories. We now find that the alkaline earthbased Ca5C60 violates this "universal" relationship; suggests the theories must include band effects.

- Theory We have shown, in a one-dimensional model of $\mathrm{Cu}$-oxide, that there exists an s-wave superconducting "instability" that is close in parameter space to a phase separation instability. The model is purely electronic with only repulsive interactions. Moreover, we observe flux-quantization consistent with a paired ground state.

Future Directions: We will continue the integrated study of synthesis, processing, and physical properties of T1bearing and other high- $T_{c}$ superconducting materials. Our goal is twofold; to understand intrinsic properties of these materials through synthesis and study of highly-crystalline bulk and thin-film samples, and to optimize, through precise control of material structure, electronic transport properties with a view toward future applications. To support this effort we have recently hired a specialist in superconducting devices. We will also continue to work on pressure effects in superoxygenated cuprates and fullerene-based and organic superconductors.

Program Quality: 3 invited publications. 18 new research publications. 4 invited presentations. Collaboratiive interactions with LANL, Oak Ridge, Argonne, NREL, Ames, AT\&T Bell Labs, DuPont, U of Wisconsin, and Indiana $\mathrm{U}$. 


\section{Physics and Chemistry of Novel Superconductors KC $020202 \&$ KC 020205}

\section{Publications}

\section{Primarily carried out under BES support at Sandia}

1. "Enhanced Vortex Pinning And Critical Current Density In Proton-Irradiated $\mathrm{YBa}_{2} \mathrm{Cu}_{3} \mathrm{O}_{6-d}$ Thin Films", E.L. Venturini, M.P. Siegal, A.E. White, S.Y. Hou, and J.M. Phillips, MRS Symp. Proc. 279, 463 (1993).

2. "Flux Quantization And Pairing One-dimensional Copper-oxidfe Models", A. Sudbo, C.M. Varma, T.Giamarchi, E.B. Stechel, and R.T. Scalettar, Phys. Rev. Lett. 70, 978 (1993).

3. "Thickness Dependence Of Electrodynamic Properties Of TICaBaCuO Thin Films", J.S. Martens, V.M. Hietala, E.L. Venturini, and W.Y. Lee. J. Appl. Phys. 73, 7571 (1993).

4. "Synthesis And Processing Of Thin Films In The T7-Ca-Ba-Cu-O System", D.S. Ginley, J.S. Martens, E.L. Venturini, C.P. Tigges, C. Ashby, and S. Volk, IEEE Trans. Appl. Supercond. 3, 1201 (1993).

5. "Di- And Tri-Carboxylic-Acid-Based Etches For Processing High-Temperature Superconducting Thin Films", D.S. Ginley, C.I.H. Ashby, T.A. Plut, D. Urea, M.P. Siegal, and J.S. Martens, Appl. Phys. Lett. 63, 2429 (1993).

6. "Superconducting Flux Flow Digital Circuits", J.S. Martens, T.E. Zipperian, V.M. Hietala, D.S. Ginley, C.P. Tigges, J.M. Phillips, and M.P. Siegal, IEEE Trans. Electron Devices 40, 656 (1993).

7. "Flux Flow Microelectronics", J.S. Martens, V.M. Hietala, T.A. Plut, D.S. Ginley, G.A. Vawter, C.P. Tigges, M.P. Siegal, J.M. Phillips, and S.Y. Hou, IEEE Trans. Appl. Supercond. 3, 2295 (1993).

8. "Pressure Dependence Of The Superconducting $\mathrm{T}_{c}$ Of Superoxygenated $\mathrm{La}_{2} \mathrm{CuO}_{4+d}$ ", J.E. Schirber, W.R. Bayless, F.C. Chou, D.C. Johnston, P.C. Canfield, Z. Fisk, Phys. Rev. B48, 6506 (1993).

9. "Neutron Diffraction On Superconducting Superoxygenated La-Sr-Cuprates", G.H. Kwei, B. Morosin, J.E. Schirber, J.A. Voigt, and E.L. Venturini, Spectroscopies for Novel Superconductors, Santa Fe, NM 3/93.

10. "Pressure Dependence Of The Superconducting Transition Of Calcium Fuilerene $\mathrm{Ca}_{5} \mathrm{C}_{60}$ ", J.E. Schirber, W.R. Bayless, A.R. Kortan, N. Kopylov, Physica C213, 190 (1993).

11. "Role Of Pressure In The Study Of Fullerenes", J.E. Schirber, G.A. Samara, B. Morosin, R.Assink, D. Loy, H. Wang, J. Williams, D. Murphy, A.R. Kortan, M. Rossiensky, O. Zhou, Q. Zhu, K. Kniaz, J.E. Fisher, Proceedings of AIRAPT/APS Joint Conference, 6/27-7/2/93 (Invited).

Collaborative - Sandia work supported by BES

12. "Correlations Between The Hall Coefficient And The Superconducting Transport Properties Of Oxygen Deficient $\mathrm{YBa}_{2} \mathrm{Cu}_{3} \mathrm{O}_{7-\mathrm{d}}$ Epitaxial Thin Films". E.C. Jones, D.K. Christen, J.R. Thompson, R. Feenstra, S. Zhu, D.H. Lowndes, J.M. Phillips, M.P. Siegal, and J.D. Budai, Phys. Rev. B47, 8986 (1993).

13. "Precipitates in $\mathrm{YBa}_{2} \mathrm{Cu}_{3} \mathrm{O}_{7-\mathrm{d}}$ Thin Films Annealed At Low Oxygen Partial Pressure", S.Y. Hou, J.M. Phillips, D.J. Werder, T.H. Tiefel, R.M. Fleming, J.H. Marshall, and M.P. Siegal, App. Phy. Let. 62, 3201 (1993).

14. "Anharmonic Effects And The Two-particle Continuum In The Raman Spectra Of $\mathrm{YBa}_{2} \mathrm{Cu}_{3} \mathrm{O}_{6.9}$, $\mathrm{TlBa}_{2} \mathrm{CaCu}_{2} \mathrm{O}_{7}$, and $\mathrm{Tl}_{2} \mathrm{Ba}_{2} \mathrm{CaCu}_{2} \mathrm{O}_{8}$ ", D. Mihailovic, K.F. McCarty, and D.S. Ginley, PRB47, 8910 (1993).

15. "NMR Study Of Local Structure In Metallic $\mathrm{La}_{2} \mathrm{CuO}_{4+d}$ ", P.C. Hammel, A.P. Reyes, S.W. Cheong, Z. Fisk, and J.E. Schirber, Phys. Rev. Letters 71, 440 (1993).

16. "Internal Magnetic Field In $\mathrm{La}_{2 \cdot x} \mathrm{Sr}_{x} \mathrm{CuO}_{4}$ :Gd Observed By EPR", C. Rettori, D. Rao, S.B. Oseroff, G. Amoretti, Z. Fisk, S-W Cheong, D. Vier, S. Schultz, M. Tovar, R.D. Zysler, and J. Schirber, PRB47, 8156 (1993).

17. "NQR Study Of Local Structure And Cooling Rate Dependent Superconductivity In $\mathrm{La}_{2} \mathrm{CuO}_{4+d}$ ", A.P. Reyes, E.T. Ahrens, P.C. Hammel, J.D. Thompson, P.C. Canfield, Z. Fisk, R.H. Heffner, and J.E. Schirber, J. Appl. Phys. 73, 6323 (1993).

18. "NMR Studies Of Oxygen-Doped $\mathrm{La}_{2} \mathrm{CuO}_{4+d}$ ", P.C. Hammel, A. Reys, E. Ahrens, D. MacLaughlin, J. Thompson, Z. Fisk, P. Canfield,. S. Cheong, and J. Schirber, Proceedings of the International Conference on Strongly Correlated Electronic Systems, 8/15/93 (Invited). 


\title{
Tailored Surfaces and Interfaces for Materials Applications KC 020202
}

\author{
Principal Investigators: G.L. Kellogg, P.J. Feibelman, N.D. Shinn, T.M. Mayer, B.S. Swartzentruber, T.A. Michalske
}

Goal: To understand the atomic-level processes that control the synthesis and processing of thin surface layers and to use this understanding to tailor the structural and electronic properties of surfaces and interfaces for specific materials applications.

\section{Recent Highlights}

- Atom Trapping by Substitutional Impurities: Direct observations with the field ion microscope show that individual $\mathrm{Rh}$ atoms are trapped in the four lattice sites adjacent to an Ir substitutional atom on the $\mathrm{Rh}(100)$ surface. Detailed investigations of the hopping rates to locations around the impurity atom have defined the energetics associated with the trap site. The strong trapping interaction confirms the suspected role of impurities in influencing the growth of single crystals and epitaxial films.

- Atom Bonding at Steps: Local Density Functional calculations show that on $\mathrm{Al}(331), \mathrm{S}$ adatoms bind preferentially at step-edge "bridge" sites. These binding sites are different than those found previously for both $\mathrm{H}$ and $\mathrm{Al}$ atoms on the same surface and also different from that for $\mathrm{S}$ atoms on close-packed $\mathrm{Al}(111)$. The calculations have clarified the roles of adatom size and valence in determining how atoms interact with steps and provided further evidence that a simple chemical picture of bonding is more appropriate near low-coordination sites on a metal surface than one might have expected.

- Interfacial Bonding Energies from Core-Level Shifts: W atoms at epitaxial Fe/W(110), Ni/W(110) and Pt/W(110) interfaces are found to exhibit discrete $W(4 f)$ core-level shifts relative to bulk $W$ atoms. The shifts are correlated to the adsorption enthalpies of the respective metals. As the first measurements to resolve this spectroscopic detail, this finding suggests a non-destructive approach to predicting adhesion energies, verifying first-principles calculations, and probing interfacial alloying. (Joint with Adhesion Science Program)

- Atomically Resolved Measurements of Nucleation During CVD: Atomically resolved scanning tunneling microscope (STM) images show specific nucleation at Si dimer sites during the initial stages of metal (Fe) CVD on $\mathrm{Si}(001)$. At low coverages, linear chains of $\mathrm{Fe}$ atoms are found to be aligned along dimer rows. Higher coverage results show evidence of decomposition on Fe nuclei, leading to three-dimensional growth of Fe clusters. These measurements are the initial results of a new effort to examine specific nucleation, surface control of nucleation, and relationship of surface dynamics to film structure and morphology.

- High-Temperature STM Measurements of Vacancy Migration and Step Rearrangement Events on Si(001): The formation and migration of vacancies on the $\mathrm{Si}(001)$ surface at elevated temperatures have been observed with our recently developed variable-temperature STM. The diffusion is highly anisotropic with the fast direction being parallel to the surface dimer rows. We have also determined the activation barrier $(-1.4 \mathrm{eV})$ for atoms to rearrange at steps on the $\mathrm{Si}(001)$ surface by measuring the step rearrangement rate as a function of temperature. Applying this real-space at-temperature probe enables us to st:dy surface kinetic processes directly, without relying on highly model dependent, after-the-fact measurements.

\section{Future Directions}

Our research will continue to focus on tailoring the properties of surfaces by the addition of thin adsorbed overlayers and on understanding the interfaces that develop when overlayers are deposited on single-crystal surfaces. A strong emphasis will be placed on how defects such as vacancies, steps, and substitutional impurities affect atom mobility and influence growth processes. This information will be used to control the growth of single-component, mixedcomponent and compositionally modulated overlayers. Combined IR and STM measurements along with controlled passivation of defect sites by $\mathrm{H}$ will be used to investigate site-specific nucleation in thermal $\mathrm{CVD}$ of organometallic precursors. New capabilities in Low Energy Electron Microscopy (LEEM) and optical techniques to examine "macroscopic" diffusion behavior will be added to enhance our real-time analysis of dynamical surface processes.

Program Quality

Co-organizers of MRS Symposia on Applications of Synchrotron Radiation Techniques to Materials Science and Surface Diffusion: Atomistics and Surface Morphology. Invited review articles in Surface Science and the MRS Bulletin. 20 Publications, 16 Invited Presentations 


\section{Tailored Surfaces and Interfaces for Materials Applications \\ KC 020202}

\section{Publications}

1. "Direct Observation Of Substitutional-atom Trapping On Metal Surfaces", G. L. Kellogg, Phys. Rev. Lett. (accepted).

2. "Diffusion Of Individual Pt Atoms On Single-Crystal Surfaces Of Rhodium", G. L. Kellogg, Phys. Rev. B 48, 11305 (1993).

3. "The Effect Of An Externally Applied Electric Field On The Diffusion Of Pt Adatoms", Dimers, And Trimers On Pt(001), G. L. Kellogg, Appl. Surface Sci. (in press).

4. "Atomic Level Studies Of Processes On Metal Surfaces", G. L. Kellogg and T. T. Tsong, (INVITED REVIEW) MRS Bulletin (in press).

5. "Energetics Of Pt Adsorption On Pt(111)", P. J. Feibelman, J. S. Nelson, and G. L. Kellogg, Phys Rev. B (submitted).

6. "Sulfur Adsorption Near A Step", P. J. Feibelman, Phys. Rev. B (submitted).

7. "Theoretical Surface Core Level Shifts For Be(0001)", P. J. Feibelman, Phys. Rev. B (submitted).

8. "Structure Of H-Covered Be(0001)", P. J. Feibelman, Phys. Rev. B 48, 11270 (1993).

9. "First-Principles Calculation Of The $\mathbf{M g}(0001)$ Surface Relaxation", A. F. Wright, P. J. Feibelman and S. R. Atlas, Surface Sci. (in press).

10. "Making Sense Of Surface Structure (Invited)", P. J. Feibelman, Chinese Journal of Physics 31, 441 (1993).

11. "Surface Diffusion: Atomistics And Surface Morphology (Summary Of Mrs Symposium B Panel Discussion)", M. H. Grabow, P. J. Feibelman, G. H. Gilmer, B. H. Cooper, and Y. W. Mo, Mat. Res. Soc. Symp. Proc 280, 11 (1993).

12. "Role Of Atomic Size And Valence In Bonding And Diffusion At Metal Surfaces", P. J. Feibelman, Mat. Res. Soc. Symp. Proc 280, 31 (1993).

13. "Making Sense Of Surface Structure (Invited Review)", P. J. Feibelman, Surface Science (in press).

14. "Relaxation Of The Clean, Cu-And H-Covered Ru(0001) Surface", P. J. Feibelman, J. E. Houston, H. L. Davis, and D. G. O'Neil, Surface Science (in press).

15. "Surface Chemistry Of $\mathrm{PH}_{3}, \mathrm{PF}_{3}$ And $\mathrm{PCl}_{3} \mathrm{ON} \mathrm{Ru(0001)",} \mathrm{H.-S.} \mathrm{Tao,} \mathrm{U.} \mathrm{Diebold,} \mathrm{T.} \mathrm{E.} \mathrm{Madey} \mathrm{and} \mathrm{N.} \mathrm{D.} \mathrm{Shinn,}$ Surface Science (in press).

16. "The Influence Of Pre-Adsorbed $\mathrm{K}$ On The Adsorption $\mathrm{Of}_{\mathrm{PF}_{3}} \mathrm{ON} \mathrm{Ru}(0001)$ Studied By Soft X-Ray Photoelectron Spectroscopy", V. Chakarian, D. Shuh, J. A. Yarmoff, H.-S. Tao, U. Diebold, B. L. Maschhoff, T. E. Madey and N. D. Shinn, J. Phys. Chem. (in press).

17. "Roughening Instability And Ion Induced Viscous Relaxation $\mathrm{Of} \mathrm{SiO}_{2}$ Surfaces". T. M. Mayer, E. Chason, A. J. Howard, J. Appl. Phys. (submitted) (Joint with Advanced Growth Program).

18. "Kinetics Of Surface Roughening And Smoothing During Ion Sputtering", E. Chason, T. M. Mayer, A. J. Howard, Proc. Mater. Res. Soc. (in press) (Joint with Advanced Growth Program).

19. "Roughening Instability And Evolution Of Ge(001) Surface During Ion Sputtering", E. Chason, T. M. Mayer, B. K. Kellerman, D. T. Mcllroy, A. J. Howard, Phys. Rev. Lett. (submitted) (Joint with Advanced Growth Program).

20. "Variable-Temperature STM Measurements Of Step Kinetics On Si(001)", N.Kitamura, B.S. Swartzentruber, M.G. Lagally, and M.B. Webb, Phys. Rev. B48, 5704 (1993). 


\title{
Boron-Rich Solids \\ KC 020205
}

\author{
Principal Investigators: T. L. Aselage, D. Emin, D. R. Tallant and P. Yang
}

Goals: To obtain an understanding of the novel properties of boron-based materials, to assess their potential for novel applications and to apply insights gained to the study of solids generally.

Highlights

- Synthesis:

- The first large $\left(1 \mathrm{~cm}^{3}\right)$ single crystals of $B_{12} P_{2}$ have been prepared by top-seeded solution growth.

-- An apparatus to deposit semiconducting boride films was designed and constructed.

- Properties:

-- Very high-temperature (300-1800 K) electrical measurements of high-quality boron carbides showed that the dependence of the conductivities on carbon concentration vanishes above $1300 \mathrm{~K}$. We infer that the very high temperature conductivity is not associated with the equilibrium density of small bipolarons. At high temperatures carriers may be induced by a glowing samples' self-radiation.

-- High-temperature contacts for our refractory borides were made with the boride conductor, $\mathrm{ZrB}_{2}$.

-- High-pressure neutron diffraction experiments yield boron carbide compressibilities that agree with those we deduced from our acoustic measurements.

-- Thermal conductivities of our P- containing boron carbides support our structural and transport models.

-- X-ray diffraction and magnetic measurements indicated that the highest $T_{c}$ 's of Mo and $\mathrm{Nb}$ diboride superconductors occur near the high-boron ends of their single-phase regions.

- Devices:

-- Refractory solid-state neutron detectors based on boron-rich insulators have been designed. An initial test using a crude design and b-boron as the boride insulator detected fluences of $10^{5}$ neutrons $/ \mathrm{cm}^{2}-\mathrm{sec}$.

- Theory:

-- Expanded calculations of group IV diborides raise $N\left(E_{F}\right)$ to bring carrier densities close to experiment.

-- Qualitative differences in their absorption bands distinquish between large and small bipolarons.

-- The polarizabilities of large bipolarons was discovered to produce a quantum-mechanical attractive force between them. Through this attraction large bipolarons can condense into a charged Bose liquid. If the liquid does not solidify, the analogy with the superfluidity of a neutral quantum Bose liquid $\left({ }^{4} \mathrm{He}\right)$ suggests that the bipolaronic liquid will be a non-BCS superconductor.

\section{Future Directions}

Crystals and films of refractory boride semiconductors will be grown and their transport and optical properties measured. The potential of the wide-gap semiconductors as high-temperature semiconductor devices and UV sensors will be evaluated. Electron microsopy studies will determine if borides' remarkable radiation tolerance extends beyond icosahedral borides. A fission cell to directly convert nuclear energy to electrical energy will be constructred with icosahedral borides to avoid the radiation damage that precludes using conventional semiconductors. In addition, simpler and potentially more sensitive neutron detectors based on the absorption of a neutron by a ${ }^{10} \mathrm{~B}$ (20\% abundant) atom in a boride semiconductor will be tested. $\mathrm{N}$ - and p-type hexaborides will be synthesized, their electronic and thermal properties studied and their usefulness in our neutron detectors and fission cells assessed.

To further develop our understanding of boron carbides' unconventional electronic transport, the dependences of the Hall mobility and Seebeck coefficients on carbon concentration will be measured on our high-quality samples. Electronic transport measurements will also resolve whether metal diborides are unconventional conductors. Electronic and thermal transport arising from a bipolaronic superconductor's collective motions will be calculated. The unusual (two-phonon) process by which phonons and large-bipolarons scatter one another is expected to result in unconventional transport.

\section{Program Quality}

- Two members of the "International Scientific Committee on Boron, Borides and Related Compounds"

- 11 invited talks (meetings and colloquia), 10 publications

- Extensive interactions with Science and Engineering Associates (SEA) and High-Z Technologies. 


\section{Boron-Rich Solids \\ KC 020205}

\section{Publications}

1. "Structure And Electronic Transport Of Undoped And Phosphorus-Doped Boron Carbides", T. L. Aselage. D. R. Tallant, D. Emin, S. B. Van Duesen and P. Yang, Japanese Journal of Applied Physics (in press).

2. "Crystal Growth Of $B_{12} P_{2}$ ", P. Yang and T. L. Aselage, Japanese Journal of Applied Physics (in press).

3. "Superconductivity Of Niobium And Molybdenum Diborides", H. Klesnar, T. L. Aselage, D. Emin, E. L. Venturini, P. P. Newcomer and B. Morosin, Japanese Journal of Applied Physics (in press).

4. "The Electronic Structure Of Transition Metal Diborides", A. C. Switendick, Japanese Journal of Applied Physics (in press).

5. "Lattice Vibrations And Electronic Transport In Group IVB Diborides", C. L. Beckel, T. J. Schilling, M. Yousef, and A. C. Switendick, Japanese Journal of Applied Physics (in press).

6. "Effects Of Disorder On Self-Trapping And Subsequent Hopping In Boron Carbides", David Emin, Japanese Journal of Applied Physics (in press).

7. “Optical Properties Of Large And Small Polarons And Bipolarons", David Emin, Physical Review B 4813691 (1993).

8. "Disorder Effects On Small-Polaron Formation And Hopping", David Emin, Proc. 5-th International Conference on Hopping and Related Phenomena, A. R. Long, editor (in press, World Scientific, London).

9. "Phonon-Mediated Attraction Between Large Bipolarons: Condensation To A Liquid", David Emin, Physical Review B 49 (in press, April 1, 1994).

10. "Phonon-Mediated Attraction Between Large Bipolarons: Condensation To A Liquid", David Emin, Physical Review Letters 721052 (1994). 


\section{CVD Sciences \\ KC 020303}

Principal Investigators: P. Esherick, M.E. Bartram, W.G. Breiland, is ol Coltrin, J.R. Creighton, P. Ho, K.P. Killeen, H.K. Moffat, J.E. Parmeter, R.J. Kee, G.H. Evans

Goals: To advance the understanding of the basic physics and chemistry of thin-film synthesis by chemical vapor deposition (CVD), including metal organic CVD, and plasma assisted CVD. This includes the development of new measurement techniques and theoretical tools that can be used both for fundamental scientific studies and for improvements of CVD processes and equipment that can be transferred to industry.

Recent Highlights:

- CRADA programs started with Motorola and EMCORE Corporations: Two independent DP-funded Technology Transfer Initiatives have evolved out of fundamental research capabilities developed under our BES program. The Motorola project will advance safer and more efficient MOCVD chemistries, whereas the EMCORE project will promote commercial applications of rotating-disk CVD technology.

- Precision measurements in well characterized CVD reactor led to new understanding of silane chemistry: We have used our rotating disk reactor to perform the most stringent test to date of a CVD chemical kinetics mechanism. Precise mapping of silicon atom densities using laser-induced fluorescence yielded the unexpected result that the $\mathrm{SiH}_{2}->\mathrm{Si}+\mathrm{H}_{2}$ reaction could not be a major source of silicon atoms.

- Surface Chemistry of Boron-Doped SiO2 CVD: We have determined that boranols on boron-doped $\mathrm{SiO}_{2}$ increase the rate of chemisorption of TEOS, an $\mathrm{SiO}_{2}$ precursor, and that the relative rate constants for TEOS reacting with boranols and silanols have dramatically different temperature dependencies. These results help explain the sensitivity of $\mathrm{SiO}_{2}$ deposition rates and growth uniformity to temperature and relative concentrati: as of boranols and silanols on the surface.

- Resistivity of Copper Films grown via CVD of (hfac)Cu(TMVS): We have achieved the lowest resistivity (1.90 $\mu \Omega \mathrm{cm})$ ever reported for an as-deposited copper film from a Cu(I) precursor. We discovered that impurities and/or defects near the copper/substrate $\left(\mathrm{Si}_{3} \mathrm{~N}_{4}\right)$ interface are the most probable cause for an increase in resistivity in very thin copper films grown via CVD from (hfac)Cu(TMVS).

- In-Situ Gas-Phase Measurements Substantiate MOCVD Methyl Radical Desorption Mechanism: Concentration profiles for $\mathrm{AsH}_{2}$ in a rotating disk GaAs MOCVD system corroborate our previous UHV surface chemistry studies and challenge the accepted theories on MOCVD pyrolysis reaction pathways. Our measurements show that methyl radicals from the decomposition of trimethylgallium desorb from the GaAs surface and recombine in the gas-phase to form methane rather than on the surface as was previously believed.

- Infrared Spectroscopy of Adsorbates on GaAs Surfaces: We have determined the structure of key adsorbates (e.g. $\mathrm{CH}_{3}, \mathrm{CH}_{2}$ ) on $\mathrm{GaAs}$ surfaces using a multiple internal reflection geometry. The $\mathrm{CH}_{3}$-surface bond orientation for the trimethylgallium dosed $\mathrm{GaAs}(100)$ surface was determined using polarization dependent infrared spectroscopy. The $\mathrm{CH}_{3}$ groups are bonded solely to surface $\mathrm{Ga}$ atoms and are tilted from the surface normal by $50 \pm 10^{\circ}$, in good agreement with the expected angle for a tetrahedral geometry.

- In situ spectral reflectance monitor for MOCVD: We have demonstrated that our normal incidence spectral reflectance technique yields accurate high-temperature optical constants. Constants extracted for AlAs and GaAs at $650 \mathrm{C}$ indicate that this robust in situ monitor of thin film growth is as precise as the more complex, and less robust ellipsometric methods.

- Surface Chemistry of $\mathrm{ZrNp} 4$ on $\mathrm{ZrC}$ : The chemistry of the metal carbide CVD precursor $\mathrm{Zr}$ (neopentyl)4 on $\mathrm{ZrC}$ thin films has been investigated. Decomposition of neopentyl groups to form methane was identified as a probable mechanism of excess carbon incorporation into metal carbide films grown with this precursor.

Future Directions:

We will pursue the development of new diagnostic tools to probe the details of deposition chemistry relevant to CVD, with our emphasis on in situ techniques to study molecular species reacting at surfaces. We will expand our knowledge base of chemical systems, including investigations of materials systems important for solar control coatings and photovoltaics. Applications of new diagnostic and computational tools will serve as the basis for developing first-principles models of the complex chemically reacting flows typical of CVD.

Program Quality:

- 1992 BES Sustained Outstanding Research Award in Materials Chemistry

- 28 publications, 25 presentations, including 10 invited talks at national or international symposia.

- Work has led to 8DP-funded CRADAs and 2 ARPA programs in past 2 years. 


\section{CVD Sciences \\ KC 020303}

\section{Publications}

1. "Comparison of Boranol and Silanol Reactivities in Boron-Doped SiO2 Chemical Vapor Deposition From Trimethyl Borate and Tetraethyl Orthosilicate", M. E. Bartram and H. K. Moffat, J. Vac. Sci. and Technol., AVS Fall Symposium, 1993. (submitted)

2. "Surface Chemistry of Boron-Doped SiO2 CVD: Enhanced Uptake of Tetraethyl Orthosilicate by Hydroxyl Groups Bonded to Boron", M. E. Bartram and H. K. Moffat, MRS Proceedings, Fall MRS Symposia, 1993. (in press)

3. "Copper CVD Chemistry on a Reactive Substrate: $\mathrm{Cu}(\mathrm{hfac}) 2$ and hfacH on Pt(111)", J. E. Parmeter, J. Phys. Chem. 97, 11530 (1993).

4. "Surface Reactions of Tetraneopentylzirconium on Zirconium Carbide Thin Films", J. E. Parmeter, D. C. Smith, and M. D. Healy, J. Vac. Sci. Technol., submitted.

5. "The Organometallic Chemical Vapor Deposition of Transition Metal Carbides: The Use of Homoleptic Alkyls", M. D. Healy, D. C. Smith, R. R. Rubiano, R. W. Springer, and J. E. Parmeter, fall 1993 MRS proceedings.

6. "In Situ Spectral Reflectance Monitoring of III-V Epitaxy", K. P. Killeen and W. G. Breiland, J. Electron. Mater. 23, 181 (1994)

7. "Spatial Detection of Gallium Atoms From Pyrolysis of Trimethylgallium in a Rotating Disk Reactor", K. P. Killeen, submitted to J. of Crystal Growth, (1994)

8. "Si Atom Density Profiles in a Rotating Disk CVD Reactor", P. Ho, W. G. Breiland, and M. E. Coltrin, Proceedings of the Twelfth International Conference on Chemical Vapor Deposition, Honolulu, Hawaii, 5/16-23/ 93.

9. "Temperature Dependence of the Reactivity of $\mathrm{OH}(\mathrm{X} 2 \Pi)$ with the Surface of Silicon Nitride and Polymer Films", E. R. Fisher, P. Ho, W. G. Brieland, and R. J. Buss, J. Phys. Chem. (submitted).

10. "Model Studies of Chemical Vapor Deposition", M. E. Coltrin, W. G. Breiland, P. Ho, Materials Technology, 8 , 250 (1993).

11. "Direct Measurement of the Reactivity of NH and OH on a Silicon Nitride Surface", R. J. Buss, P. Ho, W. G. Breiland, Proceedings of the 1993 MRS Fall Meeting, Boston, MA 11/29-12/2/93.

12. "Thermochemical Data for CVD Modeling from Ab Initio Calculations", P. Ho and Carl Melius, Proceedings of the 1993 Fall MRS Meeting, Boston, MA 11/29-12/2/93.

13. "Analysis of Diamond Growth in Subatmospheric DC Plasma-Gun Reactors", M.E. Coltrin, and D. S. Dandy, J. Appl. Phys. 74, 5803 (1993).

14. "Computational Simulation of Diamond Chemical Vapor Deposition in Premixed C2H2/O2/H2 and CH4/O2 Strained Flames", E. Meeks, R. J. Kee, M. E. Coltrin and D. S. Dandy, Combustion and Flame 92, 144 (1993).

15. "Effects of Temperature and Filament Poisoning on Diamond Growth in Hot-Filament Reactors", D. S. Dandy and M. E. Coltrin, Journal of Applied Physics, submitted.

16. "Si Atom Density Profiles in a Rotating Disk CVD Reactor", P. Ho, W. G. Breiland, and M. E. Coltrin, Proceedings of the Twelfth International Conference on Chemical Vapor Deposition, edited by K. F. Jensen and G. W. Cullen (Electrochemical Society, Pennington, NJ 1993), p.8.

17. "A Model of Elementary Chemistry and Fluid Mechanics in the Combustion of Hydrogen on Platinum Surfaces", J. Warnatz, M. D. Allendorf, R. J. Kee, and M. E. Coltrin, Combustion and Flame, (submitted 3/93).

18. "The Development and Application of Computational Simulation to Hot-Wall Multiple-Wafer Low-Pressure Chemical-Vapor-Deposition Furnaces", P.A. Spence, W. G. Houf, J. F. Grcar, R.J. Kee, R. S. Larson, W. G. Breiland, H. H. Moffat, and M. E. Coltrin, SETEC Report 93-037, 1993.

19. "Modeling the Thermal DeNox Process in Flow Reactors. Surface Effects and Nitrous Oxide Formation". P. Glarborg, K. Dam-Johansen, J. A. Miller, R. J. Kee, and M. E. Coltrin, International Journal of Chemical Kinetics, (submitted 7/93). 
20. "Interaction of Hydrogen, Methane, Ethylene, and Cyclopentane with Hot Tungsten: Implications for the Growth of Diamond Films", H. F. Winters, H. Seki, R. R. Rye and M. E. Coltrin, Journal of Applied Physics (submitted 8/93).

21. "Molecular Beam Mass Spectrometry Studies of the Chemical Vapor Deposition of Diamond", W. L. Hsu, M. C. McMaster, M. E. Coltrin and D. S. Dandy, Proceedings of the 15th Dry Processing Symposium, Tokyo, Japan, and submitted to Japanese Journal of Applied Physics (11/93).

22. "Gas-Phase Silicon Atom Densities in the Chemical Vapor Deposition of Silicon from Silane", M. E. Coltrin, W. G. Breiland, and P. Ho, Mater. Res. Soc. Symp. Proc., to be published (1994).

23. "CRESLAF (Version 4.0)*: A FORTRAN Program for Modeling Laminar, Chemically Reacting, BoundaryLayer Flow in Cylindrical or Planar Channels", M. E. Coltrin, H. K. Moffat, R. J. Kee, and F. M. Rupley, 3/1993.

24. "A Model of Elementary Chemistry and Fluid Mechanics in the Combustion of Hydrogen on Platinum Surfaces", M. D. Allendorf, R. J. Kee, J. Warnatz, and M. E. Coltrin, 4/1993.

25. "Heterogeneous Reaction Mechanisms and Kinetics Relevant to the CVD of Semiconductor Materials", J. R. Creighton and M. E. Coltrin, 1/1994.

26. "SURFTHERM: A Program to Analyze Thermochemical and Kinetic Data in Gas-Phase and Surface Chemical Reaction Mechanisms", M. E. Coltrin and H. K. Moffat, 1/1994.

27. "A Surface Kinetics Model for the Growth of Si1-xGex Films From SiH4/GeH4 Mixtures", N. Russell and W. G. Breiland, J. Appl. Phys. 73, 3525 (1993).

28. "A Model for Low Pressure Chemical Vapor Deposition in a Hot Wall Tubular Reactor", W. G. Houf,, J. F. Grcar, and W. G. Breiland, Mater. Sci. Eng. B 17, 163 (1993). 


\title{
Synthesis and Processing of Nanoclusters for Energy Applications KC 020303
}

\author{
Principal Investigators: J.P. Wilcoxon, J.E. Martin, J. Melenkiewitz, and T. Thurston.
}

Goals: The goal of this proposal is to exploit our unique micellar synthesis method to create new size-controlled metal and semiconductor nanoclusters and investigate those physical properties germane to energy applications. The most promising applications are in catalysis and photocatalysis, so we shall emphasize materials for these applications. Initially, we will produce metal clusters from base metals as candidates for replacing precious transition metals, such as platinum, for coal liquefaction and other reactions. The catalytic activity of these clusters will be evaluated in model hydrogenation reactions in collaboration with DOE technology programs on coal. We will next investigate the use of semiconductor nanoclusters to efficiently create electron-hole paris for photocatalysis. We will then bind reducing and oxidizing nanoclusters to the semiconductors to create cluster assemblies that can convert sunlight to chemical fuels. The influence of material type and structure, cluster size, surface modification, optical and dielectric properties, on the catalytic and photocatalytic properties of the cluster will be investigated. This project will directly demonstrate the integration of Basic Energy research with DOE technology development programs, especially in links with an ongoing DOE/PETC coal liquefaction program and with the solar detoxification program.

\section{Future Direction:}

(This project was very recently approved for DMS funding. The work is just getting started.)

We will synthesize catalytic homoatomic metal nanoclusters of $\mathrm{Fe}, \mathrm{Ni}, \mathrm{Co}$, and $\mathrm{SN}$ in a range of sizes and determine the cluster catalytic activity on model hydrogenation reactions. We will initiate studies of the physical properties, such as crystal phase and surface morphology, using SAD, HRTEM and STM and begin studies of their magnetic and dielectric properties as a function of size.

We will synthesize catalytic heteroclusters such as $\mathrm{Ni}-\mathrm{Mo}, \mathrm{Ni}-\mathrm{Fe}$, and $\mathrm{Ni}-\mathrm{Co}$ and determine the cluster catalytic activity on model hydrogenation reactions with the goal of improving activity by variation of the composition.

We will synthesize pure semiconductor nanoclusters for photocatalysis and measure the quantum efficiency of electron-hole creation and the recombination rate. We will synthesize photocatalytic cluster assemblies with either a reducing or oxidizing nanoelectrode and will determine the solar quantum efficiency for the half reaction as a function of cluster size and type. 


\section{Distribution}

\section{External}

Dr. Iran L. Thomas, Director (15)

Division of Materials Sciences

Office of Basic Energy Sciences

ER-13

U.S. Department of Energy

19901 Germantown Road

Germantown, MD 20874

Internal

\begin{tabular}{lll} 
MS\# & Org\# & \\
\hline 0360 & 1000 & A.R.C. Westwood \\
0320 & 1010 & P.L. Mattern \\
0335 & 1100 & F.L. Vook (5) \\
0349 & 1102 & S.T. Picraux \\
0346 & 1103 & G.A. Samara (8) \\
1056 & 1111 & B.L. Doyle \\
0350 & 1112 & S.M. Myers \\
0344 & 1114 & T.A. Michalske \\
0601 & 1126 & P. Esherick \\
0601 & 1128 & G.N. Hays \\
0345 & 1153 & D. Emin \\
0345 & 1153 & E.L. Venturini \\
0350 & 1155 & G.C. Osbourn \\
1079 & 1300 & P.S. Peercy \\
0603 & 1311 & J.Y. Tsao \\
0336 & 1700 & R.J. Eagan \\
0338 & 1703 & D.W. Schaefer \\
1349 & 1708 & R.E. Loehman \\
0337 & 1800 & A.D. Romig \\
0339 & 1802 & G.E. Pike \\
0724 & 6000 & D.L. Hartley \\
0725 & 6908 & J.S. Binkley \\
0899 & 7141 & Technical Library (5) \\
0619 & 7151 & Technical Publications (3) \\
0100 & $7613-2$ & Document Processing (10) \\
& & for DOE/OSTI \\
9163 & 8302 & W. Bauer \\
9161 & 8341 & W.G. Wolfer \\
9056 & 8355 & G.A. Fisk \\
9018 & $8523-2$ & Central Technical Files
\end{tabular}




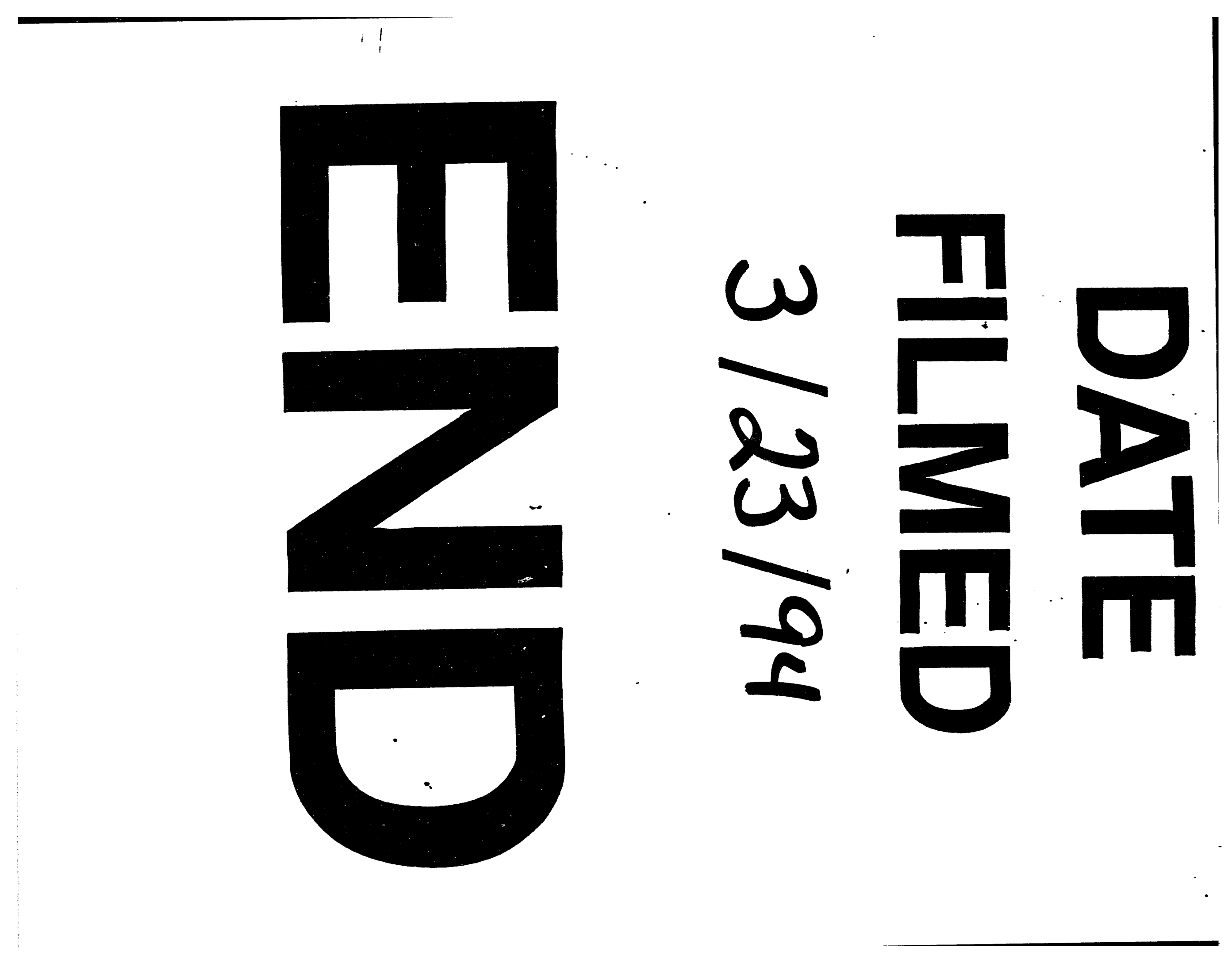




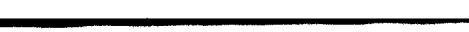

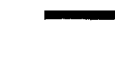

